\title{
The normal distribution is freely selfdecomposable
}

\author{
Takahiro Hasebe \\ Noriyoshi Sakuma \\ Department of Mathematics, \\ Department of Mathematics, \\ Hokkaido University \\ Aichi University of Education \\ thasebe@math.sci.hokudai.ac.jp \\ sakuma@auecc.aichi-edu.ac.jp \\ Steen Thorbjørnsen \\ Department of Mathematics, \\ University of Aarhus \\ steenth@math.au.dk
}

July 21, 2017

\begin{abstract}
The class of selfdecomposable distributions in free probability theory was introduced by BarndorffNielsen and the third named author. It constitutes a fairly large subclass of the freely infinitely divisible distributions, but so far specific examples have been limited to Wigner's semicircle distributions, the free stable distributions, two kinds of free gamma distributions and a few other examples. In this paper, we prove that the (classical) normal distributions are freely selfdecomposable. More generally it is established that the Askey-Wimp-Kerov distribution $\mu_{c}$ is freely selfdecomposable for any $c$ in $[-1,0]$. The main ingredient in the proof is a general characterization of the freely selfdecomposable distributions in terms of the derivative of their free cumulant transform.
\end{abstract}

2010 Mathematics subject classification: Primary 46L54, secondary 60E07.

Keywords: Free infinite divisibility, free selfdecomposability, free cumulant transform, normal distributions, Askey-Wimp-Kerov distributions.

\section{Introduction}

Infinitely divisible distributions and Lévy processes have constituted a major role in the development of probability theory for more than eighty years (see [22] for some main aspects). Following Voiculescu's foundation of free probability theory in the early 1980's he further introduced the class of infinitely divisible distributions with respect to free additive convolution $\boxplus$ (see $[23,9]$ ). We denote this class by $I(\boxplus)$, and refer to its members as freely infinitely divisible (FID) distributions. As in classical probability the FID distributions can be characterized as those admitting a Lévy-Khintchine representation of the free analog of the cumulant transform. This was established by Bercovici and Voiculescu in [9]. Specifically the free 
cumulant transform $\mathcal{C}_{\mu}$ of a (Borel-) probability measure $\mu$ on $\mathbb{R}$ is defined in terms of its Cauchy-Stieltjes transform $G_{\mu}$ given by

$$
G_{\mu}(z)=\int_{\mathbb{R}} \frac{1}{z-t} \mu(d t), \quad\left(z \in \mathbb{C}^{+}\right),
$$

where $\mathbb{C}^{+}$(resp. $\left.\mathbb{C}^{-}\right)$denotes the set of complex numbers with strictly positive (resp. strictly negative) imaginary part. Note in particular that $\operatorname{Im}\left(G_{\mu}(z)\right)<0$ for any $z$ in $\mathbb{C}^{+}$, and hence we may consider the reciprocal Cauchy transform $F_{\mu}: \mathbb{C}^{+} \rightarrow \mathbb{C}^{+}$given by $F_{\mu}(z)=1 / G_{\mu}(z)$ for $z$ in $\mathbb{C}^{+}$. For any probability measure $\mu$ on $\mathbb{R}$ and any $\lambda$ in $(0, \infty)$ there exist positive numbers $\alpha, \beta$ and $M$ such that $F_{\mu}$ is univalent on the set $\Gamma_{\alpha, \beta}:=\left\{z \in \mathbb{C}^{+}|\operatorname{Im}(z)>\beta,| \operatorname{Re}(z) \mid<\alpha \operatorname{Im}(z)\right\}$ and such that $F_{\mu}\left(\Gamma_{\alpha, \beta}\right) \supset \Gamma_{\lambda, M}$. Therefore the right inverse $F_{\mu}^{-1}$ of $F_{\mu}$ exists on $\Gamma_{\lambda, M}$, and the free cumulant transform $\mathcal{C}_{\mu}$ can be defined by

$$
\mathcal{C}_{\mu}(w)=w F_{\mu}^{-1}(1 / w)-1, \quad \text { for all } w \text { such that } 1 / w \in \Gamma_{\lambda, M} .
$$

The name refers to the fact that $\mathcal{C}_{\mu}$ linearizes free additive convolution (cf. [9]). Variants of $\mathcal{C}_{\mu}$ (with the same linearizing property) are the $R$-transform $\mathcal{R}_{\mu}$ and the Voiculescu transform $\varphi_{\mu}$ related by the following equalities:

$$
\mathcal{C}_{\mu}(w)=w \mathcal{R}_{\mu}(w)=w \varphi_{\mu}\left(\frac{1}{w}\right) .
$$

The free version of the Lévy-Khintchine representation now amounts to the statement that a probability measure $\mu$ on $\mathbb{R}$ is in $I(\boxplus)$, if and only if there exist $a \geqslant 0, \eta \in \mathbb{R}$ and a Lévy measure ${ }^{1} \nu$ such that

$$
\mathcal{C}_{\mu}(w)=a w^{2}+\eta w+\int_{\mathbb{R}}\left(\frac{1}{1-w x}-1-w x 1_{[-1,1]}(x)\right) \nu(d x) .
$$

The triplet $(a, \eta, \nu)$ is uniquely determined and referred to as the free characteristic triplet for $\mu$, and $\nu$ is referred to as the free Lévy measure for $\mu$. In terms of the Voiculescu transform $\varphi_{\mu}$ the free Lévy-Khintchine representation takes the form:

$$
\varphi_{\mu}(z)=\gamma+\int_{\mathbb{R}} \frac{1+t z}{z-t} \sigma(d t), \quad\left(z \in \mathbb{C}^{+}\right),
$$

where the free generating pair $(\gamma, \sigma)$ is uniquely determined and related to the free characteristic triplet by the formulas:

$$
\begin{aligned}
\nu(\mathrm{d} t) & =\frac{1+t^{2}}{t^{2}} \cdot 1_{\mathbb{R} \backslash\{0\}}(t) \sigma(\mathrm{d} t), \\
\eta & =\gamma+\int_{\mathbb{R}} t\left(1_{[-1,1]}(t)-\frac{1}{1+t^{2}}\right) \nu(\mathrm{d} t), \\
a & =\sigma(\{0\}) .
\end{aligned}
$$

In particular $\sigma$ is a finite measure. The right hand side of (4) gives rise to an analytic function defined on all of $\mathbb{C}^{+}$, and in fact the property that $\varphi_{\mu}$ can be extended analytically to all of $\mathbb{C}^{+}$also characterizes the measures in $I(\boxplus)$. More precisely Bercovici and Voiculescu established in [9] the following fundamental result:

Theorem 1.1. A probability measure $\mu$ on $\mathbb{R}$ is in $I(\boxplus)$, if and only if the Voiculescu transform $\varphi_{\mu}$ has an analytic extension defined on $\mathbb{C}^{+}$with values in $\mathbb{C}^{-} \cup \mathbb{R}$.

\footnotetext{
${ }^{1} \mathrm{~A}$ (Borel-) measure $\nu$ on $\mathbb{R}$ is called a Lévy measure, if $\nu(\{0\})=0$ and $\int_{\mathbb{R}} \min \left\{1, x^{2}\right\} \nu(d x)<\infty$.
} 
Research on FID distributions developed rapidly since 1999, when Bercovici and Pata introduced and studied a natural bijection between the classes of classically and freely infinitely divisible distributions (see [10] and [6]). As a natural step in this development the class of freely selfdecomposable (FSD) distributions was introduced in [5]. A probability distribution $\mu$ on $\mathbb{R}$ is said to be FSD, if, for any $c$ in $(0,1)$ there exists a probability measure $\rho_{c}$ such that $\mu=\mathbf{D}_{c}(\mu) \boxplus \rho_{c}$, where $\mathbf{D}_{c}(\mu)$ denotes the scaling of $\mu$ by the constant $c$. We denote the set of all freely selfdecomposable distributions by $L(\boxplus)$. Chistyakov and Goetze $[12$, Theorem 2.8] identified the class $L(\boxplus)$ with the set of possible weak limits of

$$
\delta_{a_{n}} \boxplus \mathbf{D}_{b_{n}}\left(\mu_{1} \boxplus \mu_{2} \boxplus \cdots \boxplus \mu_{n}\right), \quad n=1,2,3, \ldots,
$$

where $a_{n} \in \mathbb{R}, b_{n}>0$ and $\mu_{1}, \mu_{2}, \ldots$ are probability measures on $\mathbb{R}$ such that $\left\{\mathbf{D}_{b_{n}}\left(\mu_{k}\right)\right\}_{1 \leq k \leq n, 1 \leq n}$ forms an infinitesimal array. This is in complete analogy with the classical limit theorem for (classically) selfdecomposable distributions (see e.g. the book of Gnedenko and Kolmogorov [14]).

If $\mu$ is FSD then $\mu$ is automatically FID (see [5]), and therefore has a Lévy-Khintchine representation. The FSD distributions can then (in full analogy with selfdecomposability in classical probability) be characterized as the FID measures for which the free Lévy measure $\nu$ (appearing in the free characteristic triplet) takes the form:

$$
\nu(d x)=\frac{k(x)}{|x|} 1_{\mathbb{R} \backslash\{0\}}(x) d x,
$$

where the function $k: \mathbb{R} \backslash\{0\} \rightarrow[0, \infty)$ is non-decreasing on $(-\infty, 0)$ and non-increasing on $(0, \infty)$. From this characterization one can readily list a number of examples of FSD distributions.

Examples 1.2. (i) For any $a$ in $\mathbb{R}$ and $r$ in $(0, \infty)$ the semi-circle distribution centered at $a$ and of radius $r$ is the probability measure $\gamma_{a, r}$ given by

$$
\gamma_{a, r}(d t)=\frac{2}{\pi r^{2}} \sqrt{r^{2}-(t-a)^{2}} 1_{[a-r, a+r]}(t) d t
$$

These distributions are freely selfdecomposable, as $\gamma_{a, r}$ has free characteristic triplet $\left(\frac{r^{2}}{4}, a, 0\right)$.

(ii) The free stable distributions with index $\alpha \in(0,2)$ are FSD, as they have free characteristic triplets $(0, \eta, \nu)$, where $\nu$ has the form $(7)$ with

$$
k(x)=c x^{-\alpha} 1_{(0, \infty)}(x)+c^{\prime}|x|^{-\alpha} 1_{(-\infty, 0)}(x),
$$

and $c, c^{\prime}$ are parameters in $[0, \infty)$. The main distributional properties of the free stable distributions were uncovered by Biane in the appendix to [10].

(iii) The free Meixner distributions have been studied intensely by e.g. Saitoh and Yoshida [21], Anshelevich [2] and Bryc and Bożejko [11]. In [11] these distributions are introduced as the two-parameter family $\left\{\mu_{a, b} \mid a \in \mathbb{R}, b \geqslant-1\right\}$ of probability measures with Cauchy-Stieltjes transforms given by

$$
G_{\mu_{a, b}}(z)=\frac{(1+2 b) z+a-\sqrt{(z-a)^{2}-4(1+b)}}{2\left(b z^{2}+a z+1\right)}, \quad\left(z \in \mathbb{C}^{+}\right) .
$$

More generally all increasing affine transformations of the measures $\mu_{a, b}$ are also referred to as free Mexiner distributions. It was shown in [21] that $\mu_{a, b}$ is $\boxplus$-infinitely divisible when $b \geqslant 0$. If $a=b=0$, $\mu_{a, b}$ is a semi-circle distribution and hence FSD. The case $b=0, a \neq 0$ corresponds to the free Poisson 
distributions, which are not FSD (see (vi) below). If $b>0$, the free Lévy measure for $\mathbf{D}_{c}\left(\mu_{a, b}\right)$ is given by

$$
\nu(d x)=\frac{1}{2 \pi b} \frac{\sqrt{4 b c^{2}-(x-c a)^{2}}}{x^{2}} 1_{[c a-2 c \sqrt{b}, c a+2 c \sqrt{b}]}(x) d x
$$

for any positive number $c$. Elementary calculus shows that the function

$$
k(x)=\frac{1}{2 \pi b} \frac{\sqrt{4 b c^{2}-(x-c a)^{2}}}{|x|} 1_{[c a-2 c \sqrt{b}, c a+2 c \sqrt{b}]}(x)
$$

satisfies the monotonicity property described in (7), if and only if $4 b \geqslant a^{2}$. Thus $\mathbf{D}_{c}\left(\mu_{a, b}\right)$ is FSD, if and only if $4 b \geqslant a^{2}$. In case this inequality is strict, $\mu_{a, b}$ is termed a pure free Meixner law in [11], whereas the case $4 b=a^{2}$ is referred to as a free gamma distribution.

(iv) Pérez-Abreu and Sakuma [20] introduced another type of free gamma distributions, namely the images of the classical gamma distributions under the Bercovici-Pata bijection. They have free Lévy measure in the form:

$$
\nu(d x)=\frac{c e^{-\alpha x}}{x} 1_{(0, \infty)}(x) d x
$$

where $\alpha$ and $c$ are positive parameters. As the function $x \mapsto c e^{-\alpha x}$ is non-increasing on $(0, \infty)$, these free gamma distributions are also FSD. Their main distributional properties were uncovered by Haagerup and Thorbjørnsen in [15].

(v) The Student t-distribution with 3 degrees of freedom is the probability measure given by the Lebesgue density

$$
f(t)=\frac{2}{\pi \sqrt{3}}\left(1+\frac{t^{2}}{3}\right)^{-2}, \quad(t \in \mathbb{R}) .
$$

In the recent paper [16] it was found that this distribution is FSD.

(vi) For $\lambda$ in $(0, \infty)$ and $\alpha$ in $\mathbb{R} \backslash\{0\}$ the free Poisson distribution with parameters $(\lambda, \alpha)$ is the probability measure $\mu_{\lambda, \alpha}$ given by

$$
\mu_{\lambda, \alpha}(d t)=(1-\lambda)^{+} \delta_{0}(d t)+\frac{1}{2 \pi|\alpha| t} \sqrt{4 \lambda \alpha^{2}-(t-\alpha(1+\lambda))^{2}} 1_{\left[(1-\sqrt{\lambda})^{2},(1+\sqrt{\lambda})^{2}\right]}\left(\alpha^{-1} t\right) d t
$$

(see e.g. [19]). This distribution is FID but not FSD, since its free Lévy measure is $\nu(d t)=\lambda \delta_{\alpha}(d t)$. Note that, in some contexts, the free Poisson distributions are also referred to as free gamma distributions (not to be mistaken with the two classes described above).

The examples above illustrate the general fact that all FSD distributions are unimodal (in full analogy with classical probability theory). This was established in [17].

Triggered by a question of Pérez-Abreu, it was recently proved by Belinschi et al. (see [7]) that the classical normal (or Gaussian) distributions are FID. The proof is based on the characterization of $I(\boxplus)$ in Theorem 1.1. As a natural follow-up question Marek Bożejko asked whether the normal distributions are FSD or not. In order to answer Bożejko's question (in the positive), this paper establishes a characterization of the free cumulant transform of FSD distributions akin to Theorem 1.1 (see Theorem 2.7 below). Based on some facts about the Voiculescu transform of the normal distribution, established in [7], and a fundamental theorem due to Kerov (see Theorem 3.1), we can subsequently argue that the normal distributions satisfy this 
characterization. More generally we prove, using the same method, that the Askey-Wimp-Kerov distribution $\mu_{c}$ is FSD for any $c$ in $[-1,0]$. Let us recall here (see e.g. [18]) that for any $c$ in $(-1, \infty)$ the Askey-WimpKerov distribution $\mu_{c}$ is the measure on $\mathbb{R}$ with Lebesgue density

$$
\kappa_{c}(t)=\frac{1}{\sqrt{2 \pi} \Gamma(c+1)}\left|D_{-c}(i t)\right|^{-2}, \quad(t \in \mathbb{R}),
$$

where $D_{-c}(z)$ is the solution to the differential equation:

$$
\frac{d^{2} y}{d z^{2}}+\left(\frac{1}{2}-c-\frac{z^{2}}{4}\right) y=0
$$

satisfying the initial conditions:

$$
D_{-c}(0)=\frac{\Gamma\left(\frac{1}{2}\right) 2^{-c / 2}}{\Gamma\left(\frac{1+c}{2}\right)}, \quad \text { and } \quad D_{-c}^{\prime}(0)=\frac{\Gamma\left(-\frac{1}{2}\right) 2^{-(c+1) / 2}}{\Gamma\left(\frac{c}{2}\right)} .
$$

When $c>0$, the solution $D_{-c}$ has the integral representation

$$
D_{-c}(z)=\frac{e^{-z^{2} / 4}}{\Gamma(c)} \int_{0}^{\infty} e^{-z x} x^{c-1} e^{-x^{2} / 2} d x .
$$

It was proved in [3] that for any $c$ in $(-1, \infty)$ the measure $\mu_{c}$ is a probability measure. The case $c=0$ corresponds to the standard Gaussian distribution $N(0,1)$, and the family $\left(\mu_{c}\right)_{c \in(-1, \infty)}$ can be extended continuously at -1 by defining $\mu_{-1}$ to be the Dirac point mass $\delta_{0}$ at 0 . Then for all $c$ in $[-1, \infty)$ the Cauchy-Stieltjes transform $G_{\mu_{c}}$ has the continued fraction expansion:

$$
G_{\mu_{c}}(z)=\frac{1}{z-\frac{c+1}{z-\frac{c+2}{z-\frac{c+3}{z-\cdots}}},}
$$

or, equivalently, the orthogonal polynomials $\left(H_{n}(x ; c)\right)_{n \in \mathbb{N}_{0}}$ with respect to $\mu_{c}$ are given by the recurrence relation:

$$
H_{n+1}(x ; c)=x H_{n}(x ; c)-(c+n) H_{n-1}(x ; c), \quad(n \geqslant 1),
$$

with $H_{0}(x, c)=1$ and $H_{1}(x ; c)=x$. In the case $c=0$, one recovers the Hermite polynomials (the orthogonal polynomials with respect to $N(0,1))$, and for general $c$ the polynomials $H_{n}(x ; c)$ are referred to as associated Hermite polynomials (cf. [3]). Further information is available in $[3,7,18]$.

The remaining part of the paper is organized as follows: In Section 2 we establish the above mentioned characterization of the free cumulant transforms of FSD distributions. The proofs of some technical (but rather elementary) lemmas in this section are deferred to an appendix in order to maintain a steady flow of the paper. In Section 3, we prove the free selfdecomposability of the Askey-Wimp-Kerov distribution $\mu_{c}$ for any $c$ in $[-1,0]$, and as an immediate corollary we conclude that all normal distributions are freely selfdecomposable. 


\section{A characterization of free selfdecomposability in terms of the free cumulant transform}

In this section we establish a characterization of free selfdecomposability akin to the characterization of free infinite divisibility in Theorem 1.1. To prove this result (Theorem 2.7 below), we first need to establish some lemmas. The first four lemmas below are rather elementary, but for completeness we include proofs of Lemma 2.1, Lemma 2.3 and Lemma 2.4 in the appendix. A proof of Lemma 2.2 can be found in e.g. [13, page 150].

Throughout the paper $\log (z)$ denotes the usual (real-valued) logarithm of $z$, whenever $z$ is a positive real number. When $z$ is a complex number, the relevant branch of the logarithm will be specified, if it is not clear from the context.

Lemma 2.1. Let $a, b$ be real numbers, such that $a<b$, and let $f:[a, b] \rightarrow \mathbb{R}$ be a continuous function. Consider further the standard argument function $\arg : \mathbb{C} \backslash\{i y \mid y \in(-\infty, 0]\} \rightarrow\left(-\frac{\pi}{2}, \frac{3 \pi}{2}\right)$. Then the following assertions hold:

(i) As $v \downarrow 0$ we have that

$$
\frac{1}{2} \int_{a}^{b} f(x) \log \left((x-u)^{2}+v^{2}\right) d x \longrightarrow \int_{a}^{b} f(x) \log (|x-u|) d x \quad \text { uniformly w.r.t. } u \in[a, b] .
$$

(ii) For any anti-derivative $F$ of $f$, we have that

$$
\int_{a}^{b} f(x) \arg (u+i v-x) d x \longrightarrow i \pi(F(b)-F(u)) \quad \text { as } v \downarrow 0, \text { uniformly w.r.t. } u \in[a, b] .
$$

(iii) As $u+i v \rightarrow 0$ from $\mathbb{C}^{+}$we have that

$$
\int_{a}^{b} f(x) \log \left((u-x)^{2}+v^{2}\right) d x \longrightarrow \int_{a}^{b} f(x) \log \left(x^{2}\right) d x
$$

Lemma 2.2. Let $\rho$ be a finite Borel measure on $\mathbb{R}$, and let $a, b$ be real numbers such that $a<b$, and such that $\rho(\{a\})=\rho(\{b\})=0$. Let further $l(x)=\rho((x, \infty))$ for any $x$ in $\mathbb{R}$. Then for any $f$ in $C^{1}([a, b])$ we have that

$$
\int_{a}^{b} f(x) \rho(d x)=-[f(x) l(x)]_{a}^{b}+\int_{a}^{b} f^{\prime}(x) l(x) d x .
$$

Lemma 2.3. Let $\rho$ be a Borel measure on $\mathbb{R}$ such that $\int_{\mathbb{R}} \log (2+|x|) \rho(d x)<\infty$. Consider further the function $k: \mathbb{R} \backslash\{0\} \rightarrow[0, \infty)$ given by

$$
k(x)= \begin{cases}\int_{x}^{\infty} \frac{1+y^{2}}{y^{2}} \rho(d y), & \text { if } x>0 \\ \int_{-\infty}^{x} \frac{1+y^{2}}{y^{2}} \rho(d y), & \text { if } x<0\end{cases}
$$

Then $k$ is increasing on $(-\infty, 0)$, decreasing on $(0, \infty)$, and the following assertions hold:

(i) The measure $\frac{k(x)}{|x|} 1_{\mathbb{R} \backslash\{0\}}(x) d x$ is a Lévy measure.

(ii) $x^{2} k(x) \rightarrow 0$ as $x \rightarrow 0$.

(iii) $k(x) \log (|x|) \rightarrow 0$ as $|x| \rightarrow \infty$. 
(iv) For any $z$ in $\mathbb{C}^{-}$we have that

$$
\lim _{|x| \rightarrow \infty}\left(\log (1-x z)+\frac{x z}{1+x^{2}}\right) k(x)=0=\lim _{x \rightarrow 0}\left(\log (1-x z)+\frac{x z}{1+x^{2}}\right) k(x)
$$

where $\log$ is the standard branch of the logarithm on $\mathbb{C} \backslash(-\infty, 0]$.

Lemma 2.4. Let $a, b$ be real numbers, such that $a<b$, and let $m$ be a positive integer. Suppose further that $f:(a, b) \rightarrow \mathbb{R}$ belongs to $L^{1}((a, b), d x) \cap C^{m}((a, b))$. Consider also the Cauchy transform of $f:$

$$
G_{f}(z)=\int_{a}^{b} \frac{f(x)}{z-x} d x, \quad\left(z \in \mathbb{C}^{+}\right) .
$$

Then $G_{f}$ and all of its derivatives up to order $m-1$ can be extended to continuous functions on $\mathbb{C}^{+} \cup(a, b)$.

Lemma 2.5. Suppose that $k$ is a function in $C^{\infty}(\mathbb{R} \backslash\{0\})$ with bounded support and such that $k$ and all its derivatives are bounded functions on $\mathbb{R} \backslash\{0\}$. Suppose in addition that $k$ is increasing on $(-\infty, 0)$ and decreasing on $(0, \infty)$, and let $\mu$ be the measure in $L(\boxplus)$ with free characteristic triplet $\left(0, \int_{-1}^{1} \operatorname{sign}(t) k(t) d t, \frac{k(t)}{|t|} d t\right)$.

Then the free cumulant transform $\mathcal{C}_{\mu}$ extends to an analytic function $\mathcal{C}_{\mu}: \mathbb{C}^{-} \rightarrow \mathbb{C}$, such that $\operatorname{Im}\left(\mathcal{C}_{\mu}^{\prime}(z)\right) \leqslant$ 0 for any $z$ in $\mathbb{C}^{-}$.

Proof. For each $t$ in $\mathbb{R} \backslash\{0\}$ we put $\tilde{k}(t)=\operatorname{sign}(t) k(t)$. Since $\mu \in L(\boxplus) \subseteq I(\boxplus)$, it follows from Theorem 1.1 and (2) that $\mathcal{C}_{\mu}$ can be extended to the analytic function $\mathcal{C}_{\mu}: \mathbb{C}^{-} \rightarrow \mathbb{C}$ given by

$$
\begin{aligned}
\mathcal{C}_{\mu}(w) & =w \int_{-1}^{1} \tilde{k}(t) d t+\int_{\mathbb{R}}\left(\frac{1}{1-w t}-1-w t 1_{[-1,1]}(t)\right) \frac{k(t)}{|t|} d t=\int_{\mathbb{R}}\left(\frac{1}{1-w t}-1\right) \frac{k(t)}{|t|} d t \\
& =w \int_{\mathbb{R}} \frac{t}{1-w t} \frac{k(t)}{|t|} d t=w \int_{\mathbb{R}} \frac{\tilde{k}(t)}{1-w t} d t
\end{aligned}
$$

for any $w$ in $\mathbb{C}^{-}$. Setting $w=\frac{1}{z}$ we find for any $z$ in $\mathbb{C}^{+}$that

$$
\mathcal{C}_{\mu}\left(\frac{1}{z}\right)=\frac{1}{z} \int_{\mathbb{R}} \frac{\tilde{k}(t)}{1-\frac{t}{z}} d t=\int_{\mathbb{R}} \frac{\tilde{k}(t)}{z-t} d t=: G_{\tilde{k}}(z) .
$$

Choosing $n$ in $\mathbb{N}$ such that the support of $k$ is contained in $[-n, n]$, it follows by application of Lemma 2.4 to the restrictions of $\tilde{k}$ to $(-n, 0)$ and $(0, n)$ that $G_{\tilde{k}}$ and all its derivatives can be extended to continuous functions on $\mathbb{C}^{+} \cup(-n, 0) \cup(0, n)$. Letting $n \rightarrow \infty$, we conclude that $G_{\tilde{k}}$ and all its derivatives can be extended to continuous functions on $\mathbb{C}^{+} \cup(\mathbb{R} \backslash\{0\})$. From $(8)$ we have that

$$
\mathcal{C}_{\mu}^{\prime}\left(\frac{1}{z}\right)=-z^{2} G_{\tilde{k}}^{\prime}(z)
$$

for any $z$ in $\mathbb{C}^{+}$. In particular we thus deduce that the function $z \mapsto \mathcal{C}_{\mu}^{\prime}(1 / z)$ can be extended to a continuous function on $\mathbb{C}^{+} \cup(\mathbb{R} \backslash\{0\})$, and hence $\mathcal{C}_{\mu}^{\prime}$ can be extended to a continuous function on $\mathbb{C}^{-} \cup(\mathbb{R} \backslash\{0\})$. With $n$ chosen as above, we note further by dominated convergence that

$$
\mathcal{C}_{\mu}^{\prime}\left(\frac{1}{z}\right)=\int_{-n}^{n} \frac{z^{2}}{(z-t)^{2}} \tilde{k}(t) d t \longrightarrow \int_{-n}^{n} \tilde{k}(t) d t=\int_{\mathbb{R}} \tilde{k}(t) d t \quad \text { as }|z| \rightarrow \infty, z \in \mathbb{C}^{+} \cup \mathbb{R} .
$$

It follows thus that the function $\Psi: \mathbb{C}^{-} \cup \mathbb{R} \rightarrow \mathbb{C}$ given by

$$
\Psi(w)= \begin{cases}\mathcal{C}_{\mu}^{\prime}(w), & \text { if } w \in \mathbb{C}^{-} \cup(\mathbb{R} \backslash\{0\}), \\ \int_{\mathbb{R}} \tilde{k}(t) d t, & \text { if } w=0,\end{cases}
$$

is continuous. In addition $\operatorname{Im}(\Psi)$ is harmonic on $\mathbb{C}^{-}$. We shall argue below that 
(a) $\operatorname{Im}(\Psi(x)) \leqslant 0$ for any $x$ in $\mathbb{R}$.

(b) $\Psi(w) \rightarrow 0$, as $|w| \rightarrow \infty, w \in \mathbb{C}^{-} \cup \mathbb{R}$.

Once (a) and (b) are verified, the proof is completed as follows: Given any $\epsilon$ in $(0, \infty)$ and $w_{0}$ in $\mathbb{C}^{-}$, we choose $R$ in $(0, \infty)$, such that $R>\left|w_{0}\right|$, and such that $|\Psi(w)| \leqslant \epsilon$ for all $w$ in $\mathbb{C}^{-} \cup \mathbb{R}$ satisfying that $|w| \geqslant R$. Putting $\gamma_{R}=\left\{R e^{i \theta} \mid \theta \in[-\pi, 0]\right\}$, it follows now by the maximum principle for harmonic functions that

$$
\operatorname{Im}\left(\mathcal{C}_{\mu}^{\prime}\left(w_{0}\right)\right)=\operatorname{Im}\left(\Psi\left(w_{0}\right)\right) \leqslant \sup \left\{\operatorname{Im}(\Psi(w)) \mid w \in[-R, R] \cup \gamma_{R}\right\} \leqslant \epsilon,
$$

Since $\epsilon$ was arbitrary, we conclude that $\operatorname{Im}\left(\mathcal{C}_{\mu}^{\prime}\left(w_{0}\right)\right) \leqslant 0$, as desired.

It remains to verify (a) and (b): Regarding (a) consider a fixed number $a$ in $(0, \infty)$. Then for any $x$ in $(a, \infty)$ and any positive integer $n$ it follows from (9) that

$$
\begin{aligned}
G_{\tilde{k}}\left(x+\frac{i}{n}\right) & =G_{\tilde{k}}\left(a+\frac{i}{n}\right)+\int_{a}^{x} G_{\tilde{k}}^{\prime}\left(t+\frac{i}{n}\right) d t \\
& =G_{\tilde{k}}\left(a+\frac{i}{n}\right)-\int_{a}^{x}\left(t+\frac{i}{n}\right)^{-2} \mathcal{C}_{\mu}^{\prime}\left(\left(t+\frac{i}{n}\right)^{-1}\right) d t \underset{n \rightarrow \infty}{\longrightarrow} G_{\tilde{k}}(a)-\int_{a}^{x} t^{-2} \mathcal{C}_{\mu}^{\prime}\left(t^{-1}\right) d t,
\end{aligned}
$$

where the convergence follows e.g. by uniform continuity of $z \mapsto z^{-2} \mathcal{C}_{\mu}^{\prime}\left(z^{-1}\right)$ on $[a, x] \times(i[0,1])$. At the same time the method of Stieltjes Inversion yields for Lebesgue-almost all $x$ in $(a, \infty)$ that

$$
\tilde{k}(x)=-\frac{1}{\pi} \lim _{n \rightarrow \infty} \operatorname{Im}\left(G_{\tilde{k}}\left(x+\frac{i}{n}\right)\right)=-\frac{1}{\pi} \operatorname{Im}\left(G_{\tilde{k}}(a)\right)+\frac{1}{\pi} \int_{a}^{x} t^{-2} \operatorname{Im}\left(\mathcal{C}_{\mu}^{\prime}\left(t^{-1}\right)\right) d t
$$

Since $\tilde{k}$ is continuous, this equality actually holds for all $x$ in $(a, \infty)$, and hence we further deduce that

$$
\tilde{k}^{\prime}(x)=\frac{1}{\pi} x^{-2} \operatorname{Im}\left(\mathcal{C}_{\mu}^{\prime}\left(x^{-1}\right)\right)
$$

for all $x$ in $(a, \infty)$. Since $a$ was chosen arbitrarily in $(0, \infty),(10)$ holds for all $x$ in $(0, \infty)$ and by similar argumentation also for all $x$ in $(-\infty, 0)$. Thus for any $x$ in $\mathbb{R} \backslash\{0\}$, we conclude that $\operatorname{Im}\left(\mathcal{C}_{\mu}^{\prime}\left(\frac{1}{x}\right)\right)=\pi x^{2} \tilde{k}^{\prime}(x) \leqslant$ 0 by the definition of $\tilde{k}$ and the assumptions on $k$.

Regarding (b), we show that $\mathcal{C}_{\mu}^{\prime}\left(\frac{1}{z}\right) \rightarrow 0$ as $z \rightarrow 0, z \in \mathbb{C}^{+} \cup \mathbb{R} \backslash\{0\}$. We note initially that

$$
\mathcal{C}_{\mu}^{\prime}\left(\frac{1}{z}\right)=-z^{2} G_{\tilde{k}}^{\prime}(z)=\int_{0}^{b} \frac{z^{2}}{(z-t)^{2}} k(t) d t-\int_{-b}^{0} \frac{z^{2}}{(z-t)^{2}} k(t) d t
$$

for $z$ in $\mathbb{C}^{+}$. Moreover, the assumptions on $k$ entail the existence of the limits $k^{\prime}(0+)$ and $k^{\prime}(0-)$, since (with $b$ chosen as above)

$$
k^{\prime}(x)=-\int_{x}^{b} k^{\prime \prime}(t) d t \longrightarrow-\int_{0}^{b} k^{\prime \prime}(t) d t \quad \text { as } x \downarrow 0,
$$

and similarly

$$
k^{\prime}(x)=\int_{-b}^{x} k^{\prime \prime}(t) d t \longrightarrow \int_{-b}^{0} k^{\prime \prime}(t) d t \quad \text { as } x \uparrow 0 .
$$

The same argument ensures the existence of the limits $k^{\prime \prime}(0+)$ and $k^{\prime \prime}(0-)$. Hence, for $z=x+i y$ in $\mathbb{C}^{+}$, we can perform integration by parts twice as follows:

$$
\begin{aligned}
\int_{0}^{b} \frac{z^{2}}{(z-t)^{2}} k(t) d t & =z^{2}\left[\frac{k(t)}{z-t}\right]_{0}^{b}-z^{2} \int_{0}^{b} \frac{k^{\prime}(t)}{z-t} d t \\
& =-z k(0+)-z^{2}\left(\left[-\log (z-t) k^{\prime}(t)\right]_{0}^{b}+\int_{0}^{b} \log (z-t) k^{\prime \prime}(t) d t\right) \\
& =-z k(0+)-z^{2} \log (z) k^{\prime}(0+)-z^{2} \int_{0}^{b} \log (z-t) k^{\prime \prime}(t) d t
\end{aligned}
$$


where $\log$ is the standard branch of the logarithm on $\mathbb{C} \backslash\{i y \mid y \leq 0\}$. Here $-z k(0+)-z^{2} \log (z) k^{\prime}(0+) \rightarrow 0$, as $z \rightarrow 0, z \in \mathbb{C}^{+}$. For the last term note that $k^{\prime \prime}$ extends to a continuous function on $[0, b]$, since the limit $k^{\prime \prime}(0+)$ exists in $\mathbb{R}$ as mentioned above. Hence we can apply Lemma 2.1(iii) to establish that

$$
\begin{aligned}
\limsup _{\substack{z \rightarrow 0 \\
z \in \mathbb{C}^{+}}}\left|\int_{0}^{b} \log (z-t) k^{\prime \prime}(t) d t\right| & =\limsup _{\substack{z \rightarrow 0 \\
z \in \mathbb{C}^{+}}}\left|\frac{1}{2} \int_{0}^{b} \log \left((t-x)^{2}+y^{2}\right) k^{\prime \prime}(t) d t+i \int_{0}^{b} \arg (x-t+i y) k^{\prime \prime}(t) d t\right| \\
& \leqslant\left|\frac{1}{2} \int_{0}^{b} k^{\prime \prime}(t) \log \left(t^{2}\right) d t\right|+\left\|k^{\prime \prime}\right\|_{\infty} b \pi \\
& \leqslant \frac{1}{2}\left\|k^{\prime \prime}\right\|_{\infty} \int_{0}^{b}|\log (t)| d t+\left\|k^{\prime \prime}\right\|_{\infty} b \pi<\infty
\end{aligned}
$$

so that $z^{2} \int_{0}^{b} \log (z-t) k^{\prime \prime}(t) d t \rightarrow 0$, as $z \rightarrow 0, z \in \mathbb{C}^{+}$. We conclude that $\int_{0}^{b} \frac{z^{2}}{(z-t)^{2}} k(t) d t \rightarrow 0$ as $z \rightarrow 0$, $z \in \mathbb{C}^{+}$, and similar arguments show that $\int_{-b}^{0} \frac{z^{2}}{(z-t)^{2}} k(t) d t \rightarrow 0$ as $z \rightarrow 0, z \in \mathbb{C}^{+}$. Thus we have established that $z^{2} G_{\tilde{k}}(z) \rightarrow 0$ as $z \rightarrow 0, z \in \mathbb{C}^{+}$, and since the function $z \mapsto z^{2} G_{\tilde{k}}(z)$ is continuous on $\mathbb{C}^{+} \cup(\mathbb{R} \backslash\{0\})$, this immediately implies that the same convergence holds as $z \rightarrow 0, z \in \mathbb{C}^{+} \cup(\mathbb{R} \backslash\{0\})$.

The following lemma is a modification of Lemma 4.1 in [17]. For completeness we include a full proof in the appendix.

Lemma 2.6. Let $k: \mathbb{R} \backslash\{0\} \rightarrow[0, \infty)$ be a function which is increasing on $(-\infty, 0)$, decreasing on $(0, \infty)$ and such that $\frac{k(t)}{|t|} 1_{\mathbb{R} \backslash\{0\}}(t) d t$ is a Lévy measure. Then there exists a sequence $\left(k_{n}\right)$ of functions $k_{n}: \mathbb{R} \backslash\{0\} \rightarrow$ $[0, \infty)$ satisfying the following conditions for all $n$ in $\mathbb{N}$ :

(a) $k_{n}$ has bounded support.

(b) $k_{n} \in C^{\infty}(\mathbb{R} \backslash\{0\})$, and $k_{n}$ and all its derivatives are bounded functions.

(c) $k_{n}$ is increasing on $(-\infty, 0)$ and decreasing on $(0, \infty)$.

(d) $\frac{|t| k_{n}(t)}{1+t^{2}} d t \stackrel{\mathrm{w}}{\longrightarrow} \frac{|t| k(t)}{1+t^{2}} d t \quad$ as $n \rightarrow \infty$.

With the preceding lemmas in place we are now ready to prove the following characterization of the freely selfdecomposable distributions on $\mathbb{R}$.

Theorem 2.7. For a probability measure $\mu$ on $\mathbb{R}$ the following statements are equivalent:

(i) $\mu \in L(\boxplus)$.

(ii) The free cumulant transform $\mathcal{C}_{\mu}$ of $\mu$ extends to an analytic map $\mathcal{C}_{\mu}: \mathbb{C}^{-} \rightarrow \mathbb{C}$, satisfying that $\operatorname{Im}\left(\mathcal{C}_{\mu}^{\prime}(w)\right) \leqslant 0$ for any $w \in \mathbb{C}^{-}$.

(iii) There exists $\xi$ in $\mathbb{R}$ and a measure $\rho$ on $\mathbb{R}$, satisfying that $\int_{\mathbb{R}} \log (|x|+2) \rho(d x)<\infty$, such that $\mathcal{C}_{\mu}^{\prime}$ can be extended to all of $\mathbb{C}^{-}$via the formula:

$$
\mathcal{C}_{\mu}^{\prime}(w)=\xi+\int_{\mathbb{R}} \frac{x+w}{1-x w} \rho(d x), \quad\left(w \in \mathbb{C}^{-}\right) .
$$


If (i)-(iii) are satisfied, then the pair $(\xi, \rho)$ in (iii) is unique, and the free characteristic triplet for $\mu$ is given by $\left(a, \eta, \frac{k(x)}{|x|} d x\right)$, where

$$
\begin{aligned}
a & =\frac{1}{2} \rho(\{0\}), \\
\eta & =\xi+\int_{\mathbb{R}} x\left(1_{[-1,1]}(x)-\frac{1-x^{2}}{\left(1+x^{2}\right)^{2}}\right) \frac{k(x)}{|x|} d x, \\
k(x) & = \begin{cases}\int_{x}^{\infty} \frac{1+y^{2}}{y^{2}} \rho(d y), & \text { if } x>0, \\
\int_{-\infty}^{x} \frac{1+y^{2}}{y^{2}} \rho(d y), & \text { if } x<0 .\end{cases}
\end{aligned}
$$

Remark 2.8. It is a bit unexpected that the condition (ii) implies in particular that $\mu \in I(\boxplus)$ and hence the condition in Theorem 1.1: $\operatorname{Im}\left(\varphi_{\mu}(z)\right) \leq 0$ for all $z$ in $\mathbb{C}^{+}$. We provide an interpretation of this implication in terms of free cumulants in Remark 2.11.

Proof of Theorem 2.7. (i) $\Rightarrow$ (ii): Assume that $\mu \in L(\boxplus)$ with free characteristic triplet $\left(\eta, a, \frac{k(x)}{|x|} d x\right)$, where $k$ is increasing on $(-\infty, 0)$ and decreasing on $(0, \infty)$. Then note that (cf. (3))

$$
\operatorname{Im}\left(\mathcal{C}_{\mu}^{\prime}(w)\right)=2 a \operatorname{Im}(w)+\operatorname{Im}\left(\frac{d}{d w} \int_{\mathbb{R}}\left(\frac{1}{1-t w}-1-t w 1_{[-1,1]}(t)\right) \frac{k(t)}{|t|} d t\right), \quad\left(w \in \mathbb{C}^{-}\right) .
$$

Since $a \operatorname{Im}(w) \leqslant 0$ for any $w$ in $\mathbb{C}^{-}$, we may assume without loss of generality that $a=0$. Furthermore, since the right hand side of (13) does not depend on $\eta$, it suffices to show that there exists a real constant $\eta_{0}$, such that $\operatorname{Im}\left(\mathcal{C}_{\mu_{0}}^{\prime}(w)\right) \leqslant 0$ for all $w$ in $\mathbb{C}^{-}$, where $\mu_{0}$ is the measure with free characteristic triplet $\left(0, \eta_{0}, \frac{k(t)}{|t|} d t\right)$.

By Lemma 2.6 we can choose a sequence $\left(k_{n}\right)_{n \in \mathbb{N}}$ of functions satisfying conditions (a)-(c) of that lemma, and such that $\sigma_{n}(d t) \rightarrow \sigma(d t)$ weakly as $n \rightarrow \infty$, where $\sigma_{n}(d t)=\frac{|t| k_{n}(t)}{1+t^{2}} d t$ and $\sigma(d t)=\frac{|t| k(t)}{1+t^{2}} d t$. Then let $\mu_{n}$ and $\mu_{0}$ be the measures in $L(\boxplus)$ with free generating pairs (cf. (4) and (5)) $\left(0, \sigma_{n}\right)$ and $(0, \sigma)$, respectively. For any fixed $z$ in $\mathbb{C}^{+}$we then have that

$$
\varphi_{\mu_{n}}(z)=\int_{\mathbb{R}} \frac{1+t z}{z-t} \sigma_{n}(d t) \underset{n \rightarrow \infty}{\longrightarrow} \int_{\mathbb{R}} \frac{1+t z}{z-t} \sigma(d t)=\varphi_{\mu_{0}}(z)
$$

and that

$$
\varphi_{\mu_{n}}^{\prime}(z)=-\int_{\mathbb{R}} \frac{1+t^{2}}{(z-t)^{2}} \sigma_{n}(d t) \underset{n \rightarrow \infty}{\longrightarrow}-\int_{\mathbb{R}} \frac{1+t^{2}}{(z-t)^{2}} \sigma(d t)=\varphi_{\mu_{0}}^{\prime}(z),
$$

as the functions $t \mapsto \frac{1+t z}{z-t}$ and $t \mapsto \frac{1+t^{2}}{(z-t)^{2}}$ are both continuous and bounded on $\mathbb{R}$. This further implies that

$$
\mathcal{C}_{\mu_{n}}^{\prime}\left(\frac{1}{z}\right)=\varphi_{\mu_{n}}(z)-z \varphi_{\mu_{n}}^{\prime}(z) \underset{n \rightarrow \infty}{\longrightarrow} \mathcal{C}_{\mu_{0}}^{\prime}\left(\frac{1}{z}\right)
$$

for any $z$ in $\mathbb{C}^{+}$. By Lemma 2.5 we have that $\operatorname{Im}\left(\mathcal{C}_{\mu_{n}}^{\prime}(w)\right) \leqslant 0$ for any $w$ in $\mathbb{C}^{-}$and $n$ in $\mathbb{N}$, and hence also $\operatorname{Im}\left(\mathcal{C}_{\mu_{0}}^{\prime}(w)\right) \leqslant 0$ for any $w$ in $\mathbb{C}^{-}$. Since $\mu_{0}$ has free characteristic triplet $\left(0, \eta_{0}, \frac{k(t)}{|t|}\right)$ for some real constant $\eta_{0}$, we have established the necessary condition described above.

(ii) $\Rightarrow$ (iii): Assume that (ii) is satisfied. Then the function $z \mapsto-\mathcal{C}_{\mu}^{\prime}\left(\frac{1}{z}\right)$ is analytic from $\mathbb{C}^{+}$into $\mathbb{C}^{+} \cup \mathbb{R}$, and hence by Nevanlinna-Pick representation (see e.g. [1, Formula (3.3)]) there exist $c$ in $[0, \infty), \xi$ in $\mathbb{R}$ and a finite measure $\rho$ on $\mathbb{R}$ such that

$$
-\mathcal{C}_{\mu}^{\prime}\left(\frac{1}{z}\right)=c z-\xi+\int_{\mathbb{R}} \frac{1+x z}{x-z} \rho(d x), \quad\left(z \in \mathbb{C}^{+}\right) .
$$

Then

$$
\mathcal{C}_{\mu}^{\prime}(w)=-\frac{c}{w}+\xi+\int_{\mathbb{R}} \frac{x+w}{1-x w} \rho(d x), \quad\left(w \in \mathbb{C}^{-}\right),
$$


and it remains to establish that $c=0$ and that $\int_{\mathbb{R}} \log (|x|+2) \rho(d x)<\infty$. For $y$ in $(0, \infty)$ we note that

$$
\mathcal{C}_{\mu}(-i y)=\mathcal{C}_{\mu}(-i)-i \int_{1}^{y} \mathcal{C}_{\mu}^{\prime}(-i t) d t
$$

so that

$$
\begin{aligned}
\operatorname{Re}\left(\mathcal{C}_{\mu}(-i y)\right) & =\operatorname{Re}\left(\mathcal{C}_{\mu}(-i)\right)+\operatorname{Im}\left(\int_{1}^{y} \mathcal{C}_{\mu}^{\prime}(-i t) d t\right) \\
& =\operatorname{Re}\left(\mathcal{C}_{\mu}(-i)\right)-\int_{1}^{y}\left(\frac{c}{t}+\int_{\mathbb{R}} \frac{t\left(1+x^{2}\right)}{1+t^{2} x^{2}} \rho(d x)\right) d t \\
& =\operatorname{Re}\left(\mathcal{C}_{\mu}(-i)\right)+c \log \left(\frac{1}{y}\right)+\int_{\mathbb{R}}\left(\int_{y}^{1} \frac{t\left(1+x^{2}\right)}{1+t^{2} x^{2}} d t\right) \rho(d x) \\
& =\operatorname{Re}\left(\mathcal{C}_{\mu}(-i)\right)+c \log \left(\frac{1}{y}\right)+\frac{1}{2} \int_{\mathbb{R}} \frac{1+x^{2}}{x^{2}} \log \left(\frac{1+x^{2}}{1+x^{2} y^{2}}\right) \rho(d x) .
\end{aligned}
$$

Since $\varphi_{\mu}(i v)=o(v)$ as $v \rightarrow \infty$ (see Bercovici-Voiculescu [9, Proposition 5.6]), it follows that $\lim _{y \downarrow 0} \mathcal{C}_{\mu}(-i y)=$ $-\lim _{y \downarrow 0} i y \varphi\left(i y^{-1}\right)=0$. On the other hand the monotone convergence theorem yields that

$$
\lim _{y \downarrow 0} \int_{\mathbb{R}} \frac{1+x^{2}}{x^{2}} \log \left(\frac{1+x^{2}}{1+x^{2} y^{2}}\right) \rho(d x)=\int_{\mathbb{R}} \frac{1+x^{2}}{x^{2}} \log \left(1+x^{2}\right) \rho(d x) \in[0, \infty] .
$$

We thus conclude that

$$
0=\operatorname{Re}\left(\mathcal{C}_{\mu}(-i)\right)+c \cdot \infty+\int_{\mathbb{R}} \frac{1+x^{2}}{x^{2}} \log \left(1+x^{2}\right) \rho(d x) .
$$

As a result, we obtain that $c=0$ and $\int_{\mathbb{R}} \log (|x|+2) \rho(d x)<\infty$, as desired.

(iii) $\Rightarrow$ (i): Assume that (iii) holds, and note initially that this implies that $\mathcal{C}_{\mu}$ also extends to an analytic function on all of $\mathbb{C}^{-}$. Next let $2 a=\rho(\{0\})$ and let $z$ be a fixed point in $\mathbb{C}^{-}$. Then we denote by $[-i, z]$ the straight line from $-i$ to $z$, and by $\int_{-i}^{z} \mathcal{C}_{\mu}^{\prime}(\omega) d \omega$ the path integral of $\mathcal{C}_{\mu}^{\prime}$ along $[-i, z]$. Since $z,-i \in \mathbb{C}^{-}$, it is standard to check that

$$
\sup \left\{\left|\frac{x+\omega}{1-x \omega}\right| \mid x \in \mathbb{R}, \omega \in[-i, z]\right\}<\infty,
$$

and hence we may apply Fubini's Theorem in the following calculation:

$$
\begin{aligned}
\mathcal{C}_{\mu}(z) & =\mathcal{C}_{\mu}(-i)+\int_{-i}^{z} \mathcal{C}_{\mu}^{\prime}(\omega) d \omega \\
& =\mathcal{C}_{\mu}(-i)+\int_{-i}^{z}\left(\xi+\int_{\mathbb{R}} \frac{x+\omega}{1-x \omega} \rho(d x)\right) d \omega \\
& =\mathcal{C}_{\mu}(-i)+\int_{-i}^{z}\left(\xi+2 a \omega+\int_{\mathbb{R} \backslash\{0\}} \frac{x+\omega}{1-x \omega} \rho(d x)\right) d \omega \\
& =\mathcal{C}_{\mu}(-i)+\xi(z+i)+a\left(z^{2}-i^{2}\right)+\int_{\mathbb{R} \backslash\{0\}}\left(\int_{-i}^{z} \frac{x+\omega}{1-x \omega} d \omega\right) \rho(d x) \\
& =\mathcal{C}_{\mu}(-i)+i \xi+a+\xi z+a z^{2}+\int_{\mathbb{R} \backslash\{0\}}\left[\left(-\log (1-x \omega)-\frac{x \omega}{1+x^{2}}\right)\right]_{\omega=-i}^{\omega=z} \frac{1+x^{2}}{x^{2}} \rho(d x),
\end{aligned}
$$

where $\log$ is the standard branch of the logarithm on $\mathbb{C} \backslash(-\infty, 0]$. By second order Taylor expansion, it follows that $\log (1-\omega x)=-\omega x-\frac{1}{2} \omega^{2} x^{2}+o\left(x^{2}\right)$, and therefore

$$
\log (1-\omega x)+\frac{x \omega}{1+x^{2}}=-\frac{\omega x^{3}}{1+x^{2}}-\frac{1}{2} \omega^{2} x^{2}+o\left(x^{2}\right), \quad \text { as } x \rightarrow 0,
$$

for each fixed $\omega$ in $\mathbb{C}^{-}$. This implies that

$$
\int_{[-1,1] \backslash\{0\}}\left|\log (1+x i)-\frac{x i}{1+x^{2}}\right| \frac{1+x^{2}}{x^{2}} \rho(d x)<\infty,
$$


and since also

$$
\begin{aligned}
\int_{\mathbb{R} \backslash[-1,1]}\left|\log (1+x i)-\frac{x i}{1+x^{2}}\right| \frac{1+x^{2}}{x^{2}} \rho(d x) & \leqslant \int_{\mathbb{R} \backslash[-1,1]} 2|\log (1+x i)| \rho(d x)+\int_{\mathbb{R} \backslash[-1,1]} \frac{1}{|x|} \rho(d x) \\
& \leqslant \int_{\mathbb{R} \backslash[-1,1]} 2(\log (1+|x|)+\pi) \rho(d x)+\rho(\mathbb{R} \backslash[-1,1])<\infty,
\end{aligned}
$$

by the assumptions on $\rho$, it follows that the integral $\int_{\mathbb{R} \backslash\{0\}}\left(\log (1+x i)-\frac{x i}{1+x^{2}}\right) \frac{1+x^{2}}{x^{2}} \rho(d x)$ is a well-defined complex number. We thus conclude that

$$
\mathcal{C}_{\mu}(z)=A+\xi z+a z^{2}+\int_{\mathbb{R} \backslash\{0\}}\left(-\log (1-x z)-\frac{x z}{1+x^{2}}\right) \frac{1+x^{2}}{x^{2}} \rho(d x),
$$

where $A=\mathcal{C}_{\mu}(-i)+i \xi+a+\int_{\mathbb{R} \backslash\{0\}}\left(\log (1+x i)-\frac{x i}{1+x^{2}}\right) \frac{1+x^{2}}{x^{2}} \rho(d x)$.

Now we put

$$
k(x):= \begin{cases}\int_{x}^{\infty} \frac{1+y^{2}}{y^{2}} \rho(d y), & \text { if } x>0 \\ \int_{-\infty}^{x} \frac{1+y^{2}}{y^{2}} \rho(d y), & \text { if } x<0 .\end{cases}
$$

Then for any continuity points $r, s$ of $\rho$, such that $0<r<s$, we find by application of Lemma 2.2 that

$$
\begin{aligned}
\int_{r}^{s} & \left(-\log (1-x z)-\frac{x z}{1+x^{2}}\right) \frac{1+x^{2}}{x^{2}} \rho(d x) \\
& =\left[\left(\log (1-x z)+\frac{x z}{1+x^{2}}\right) k(x)\right]_{r}^{s}+\int_{r}^{s}\left(\frac{z}{1-x z}-z \frac{d}{d x}\left(\frac{x}{1+x^{2}}\right)\right) k(x) d x \\
& =\left[\left(\log (1-x z)+\frac{x z}{1+x^{2}}\right) k(x)\right]_{r}^{s}+\int_{r}^{s}\left(\frac{x z}{1-x z}-z \frac{x-x^{3}}{\left(1+x^{2}\right)^{2}}\right) \frac{k(x)}{x} d x .
\end{aligned}
$$

Here Lemma 2.3(iv) entails that

$$
\left[\left(\log (1-x z)+\frac{x z}{1+x^{2}}\right) k(x)\right]_{r}^{s} \longrightarrow 0, \quad \text { as } r \downarrow 0 \text { and } s \uparrow \infty .
$$

Note further that

$$
\int_{r}^{s}\left(\frac{x z}{1-x z}-z \frac{x-x^{3}}{\left(1+x^{2}\right)^{2}}\right) \frac{k(x)}{x} d x=\int_{r}^{s}\left(\frac{1}{1-x z}-1-x z 1_{[-1,1]}(x)\right) \frac{k(x)}{x} d x+\int_{r}^{s} g_{z}(x) \frac{k(x)}{x} d x,
$$

where

$$
g_{z}(x)=\frac{z x}{1-x z}-\frac{1}{1-x z}+1+x z 1_{[-1,1]}(x)-z \frac{x-x^{3}}{\left(1+x^{2}\right)^{2}}=z x\left(1_{[-1,1]}(x)-\frac{1-x^{2}}{\left(1+x^{2}\right)^{2}}\right) .
$$

Since $g_{z}(x) \rightarrow 0$ as $|x| \rightarrow \infty$ and $g_{z}(x)=o\left(x^{2}\right)$ as $x \rightarrow 0$, and since $\frac{k(x)}{x} d x$ is a Lévy measure (cf. Lemma 2.3), it follows that $\int_{0}^{\infty}\left|g_{z}(x)\right| \frac{k(x)}{x} d x<\infty$.

Considering now sequences $\left(r_{n}\right)$ and $\left(s_{n}\right)$ of continuity points for $\rho$, such that $r_{n} \rightarrow 0$ and $s_{n} \rightarrow \infty$ as $n \rightarrow \infty$, it follows by combining (16)-(18) that

$$
\begin{aligned}
\int_{0}^{\infty}(- & \left.\log (1-x z)-\frac{x z}{1+x^{2}}\right) \frac{1+x^{2}}{x^{2}} \rho(d x) \\
& =\lim _{n \rightarrow \infty} \int_{r_{n}}^{s_{n}}\left(-\log (1-x z)-\frac{x z}{1+x^{2}}\right) \frac{1+x^{2}}{x^{2}} \rho(d x) \\
& =z \int_{0}^{\infty} x\left(1_{[-1,1]}(x)-\frac{1-x^{2}}{\left(1+x^{2}\right)^{2}}\right) \frac{k(x)}{x} d x+\int_{0}^{\infty}\left(\frac{1}{1-x z}-1-x z 1_{[-1,1]}(x)\right) \frac{k(x)}{x} d x .
\end{aligned}
$$


By similar arguments, it follows that

$$
\begin{aligned}
& \int_{-\infty}^{0}\left(-\log (1-x z)-\frac{x z}{1+x^{2}}\right) \frac{1+x^{2}}{x^{2}} \rho(d x) \\
& \quad=z \int_{-\infty}^{0} x\left(1_{[-1,1]}(x)-\frac{1-x^{2}}{\left(1+x^{2}\right)^{2}}\right) \frac{k(x)}{|x|} d x+\int_{-\infty}^{0}\left(\frac{1}{1-x z}-1-x z 1_{[-1,1]}(x)\right) \frac{k(x)}{|x|} d x
\end{aligned}
$$

and combining these two formulas with (15), we obtain the expression:

$$
\mathcal{C}_{\mu}(z)=A+\eta z+a z^{2}+\int_{\mathbb{R}}\left(\frac{1}{1-x z}-1-x z 1_{[-1,1]}(x)\right) \frac{k(x)}{|x|} d x
$$

where $\eta=\xi+\int_{\mathbb{R}} x\left(1_{[-1,1]}(x)-\frac{1-x^{2}}{\left(1+x^{2}\right)^{2}}\right) \frac{k(x)}{|x|} d x$. Finally, let $\mu^{\prime}$ be the measure in $L(\boxplus)$ with free characteristic triplet $\left(a, \eta, \frac{k(x)}{|x|} d x\right)$. Then by two applications of $\left[9\right.$, Proposition 5.6] we find that $0=\lim _{y \uparrow 0} \mathcal{C}_{\mu}(i y)=$ $A+\lim _{y \uparrow 0} \mathcal{C}_{\mu^{\prime}}(i y)=A$. Thus $\mu=\mu^{\prime} \in L(\boxplus)$, and this completes the proof.

Before stating the following corollary to Theorem 2.7 we recall that for a compactly supported probability measure $\mu$ on $\mathbb{R}$ the $R$-transform $\mathcal{R}_{\mu}$ can be extended analytically to an open neighborhood of 0 . Thus $\mathcal{C}_{\mu}(z)=z \mathcal{R}_{\mu}(z)$ admits a power series expansion:

$$
\mathcal{C}_{\mu}(z)=\sum_{n=1}^{\infty} \kappa_{n}(\mu) z^{n}
$$

in a ball around 0 , and the coefficients $\left\{\kappa_{n}(\mu) \mid n \geq 1\right\}$ are the free cumulants of $\mu$ (see e.g. [8]). For a general measure $\mu$ on $\mathbb{R}$ with moments of all orders the free cumulants are defined from the moments via Möbius inversion (see [19]) and (20) only holds as an asymptotic expansion (see [8]). Recall that a sequence $\left\{a_{n}\right\}_{n=1}^{\infty}$ of real numbers is said to be conditionally positive definite if the $N \times N$ matrix $\left\{a_{i+j}\right\}_{i, j=1}^{N}$ is positive definite for any $N \geq 1$ (see [19]).

Corollary 2.9. Let $\mu$ be a Borel probability measure on $\mathbb{R}$ with moments of all orders, and let $\left\{\kappa_{n}(\mu)\right\}_{n=1}^{\infty}$ be the free cumulant sequence of $\mu$. Then the following statements hold:

(i) If $\mu$ is FSD then $\left\{n \kappa_{n}(\mu)\right\}_{n=1}^{\infty}$ is conditionally positive definite.

(ii) Suppose further that $\mu$ has compact support. Then $\mu$ is FSD if and only if $\left\{n \kappa_{n}(\mu)\right\}_{n=1}^{\infty}$ is conditionally positive definite.

Remark 2.10. A Borel probability measure $\mu$ on $\mathbb{R}$ with finite moments of all orders is compactly supported if and only if the sequence $\left\{\kappa_{n}(\mu)\right\}_{n=1}^{\infty}$ does not grow faster than exponentially; i.e., there exists $c>0$ such that $\left|\kappa_{n}(\mu)\right| \leq c^{n}$ for all $n \geq 1$. This is also equivalent to the property that the Lévy measure of $\mu$ is compactly supported. See [19, Lemma 13.13, Proposition 13.15].

Remark 2.11. Suppose that $\mu$ is compactly supported. It is well known that $\mu$ is in $I(\boxplus)$ if and only if $\left\{\kappa_{n}(\mu)\right\}_{n=1}^{\infty}$ is conditionally positive definite (see e.g. [19, Theorem 13.16]). Our result then shows the implication

$$
\left\{n \kappa_{n}(\mu)\right\}_{n=1}^{\infty} \text { is conditionally positive definite } \Longrightarrow\left\{\kappa_{n}(\mu)\right\}_{n=1}^{\infty} \text { is conditionally positive definite. }
$$

This implication can be proved more directly from the following two facts: The sequence $\left\{\frac{1}{n}\right\}_{n=1}^{\infty}$ is conditionally positive definite since $\frac{1}{n}$ is the $(n-1)$-th moment of the uniform distribution on $(0,1)$; the product of two conditionally positive definite sequences is again conditionally positive definite. 
Proof of Corollary 2.9. (i) From Theorem 1.3 in [8], the asymptotic expansion of the free cumulant transform exists up to any order, and then according to Lemma A.1 in [8], the equation

$$
\mathcal{C}_{\mu}^{\prime}(z)=\sum_{n=0}^{\infty}(n+1) \kappa_{n+1}(\mu) z^{n} .
$$

holds in the sense of an asymptotic expansion. Applying the second part of [1, Theorem 3.2.1] to the function $-\mathcal{C}_{\mu}^{\prime}(1 / z)+\kappa_{1}(\mu)$ (which maps $\mathbb{C}^{+}$into $\mathbb{C}^{+} \cup \mathbb{R}$ by Theorem 2.7) then implies that the sequence $\left\{(n+2) \kappa_{n+2}(\mu)\right\}_{n=0}^{\infty}$ is a moment sequence of a finite measure.

(ii) The sufficiency is already proved in (i), so it suffices to show the necessity. This proof is similar to the discussion in [19, Chapter 13]. Suppose that $\left\{n \kappa_{n}(\mu)\right\}_{n=1}^{\infty}$ is conditionally positive definite. Since $\left\{n \kappa_{n}(\mu)\right\}_{n \geq 1}$ does not grow faster than exponentially, Proposition 13.14 in [19] yields the existence of a finite measure $\widetilde{\rho}$ on $\mathbb{R}$ with compact support such that

$$
(n+2) \kappa_{n+2}(\mu)=\int_{\mathbb{R}} x^{n} \widetilde{\rho}(d x), \quad(n \geq 0) .
$$

Therefore, for all $z$ with sufficiently small absolute value, we have that (cf. (20))

$$
\begin{aligned}
\mathcal{C}_{\mu}^{\prime}(z) & =\kappa_{1}(\mu)+\sum_{n=1}^{\infty}(n+1) \kappa_{n+1}(\mu) z^{n}=\kappa_{1}(\mu)+\sum_{n=1}^{\infty}\left(\int_{\mathbb{R}} x^{n-1} \widetilde{\rho}(d x)\right) z^{n} \\
& =\kappa_{1}(\mu)+\sum_{n=1}^{\infty}\left(\int_{\mathbb{R}}\left(x^{n+1}+x^{n-1}\right) \frac{\widetilde{\rho}(d x)}{1+x^{2}}\right) z^{n} . \\
& =\kappa_{1}(\mu)-\int_{\mathbb{R}} x \rho(d x)+\sum_{n=0}^{\infty} \int_{\mathbb{R}} x^{n+1} z^{n} \rho(d x)+\sum_{n=0}^{\infty} \int_{\mathbb{R}} x^{n} z^{n+1} \rho(d x) \\
& =\kappa_{1}(\mu)-\int_{\mathbb{R}} x \rho(d x)+\int_{\mathbb{R}} \frac{x+z}{1-x z} \rho(d x),
\end{aligned}
$$

where we put $\rho(d x)=\frac{\widetilde{\rho}(d x)}{1+x^{2}}$. From the resulting expression of this calculation it follows that $\mathcal{C}_{\mu}^{\prime}$ extends to an analytic function on $\mathbb{C}^{-}$, and hence a correctly chosen anti-derivative of $\mathcal{C}_{\mu}^{\prime}$ is an analytic extension of $\mathcal{C}_{\mu}$ to all $\mathbb{C}^{-}$. Since $\mathcal{C}_{\mu}^{\prime}$ has the form (11) it follows from Theorem 2.7 that $\mu$ is freely selfdecomposable.

\section{Free selfdecomposability of the normal distribution}

In this section we prove that the classical normal (or Gaussian) distributions belong to the class $L(\boxplus)$ of freely selfdecomposable probability distributions, and more generally that the Askey-Wimp-Kerov distributions $\mu_{c}$ belong to $L(\boxplus)$ for all $c$ in $[-1,0]$. Apart from Theorem 2.7 the proof is based on results from Belinschi et al. [7] and the following Theorem due to Kerov (see [18, Theorem 8.2.5]).

Theorem 3.1. For any $c$ in $(-1, \infty)$ there exists a probability measure $\tau_{c}$ on $\mathbb{R}$, such that the following relation holds between the Cauchy-Stieltjes transforms:

$$
-\frac{d}{d z} \log G_{\mu_{c}}(z)=G_{\tau_{c}}(z), \quad\left(z \in \mathbb{C}^{+}\right) .
$$

As a final preparation we introduce the class $\mathcal{U I}$ consisting of those Borel probability measures on $\mathbb{R}$ for which there exists a simply connected domain $\Omega$ in $\mathbb{C}$, such that $\Omega \supset \mathbb{C}^{+}$and such that the reciprocal Cauchy-Stieltjes transform $F_{\mu}$ can be extended to an analytic bijection $F_{\mu}: \Omega \rightarrow \mathbb{C}^{+}$. If $\mu$ is in $\mathcal{U I}$, then it is FID, as was proved in [4]. For distributions in $\mathcal{U I}$ Theorem 2.7 then yields the following characterization of free selfdecomposability: 
Lemma 3.2. Let $\mu$ be a measure in $\mathcal{U I}$ with domain $\Omega$ as described above. Then the following statements are equivalent:

(i) $\mu \in L(\boxplus)$.

(ii) $\operatorname{Im}\left(\omega-\frac{F_{\mu}(\omega)}{F_{\mu}^{\prime}(\omega)}\right) \leqslant 0$ for all $\omega$ in $\Omega$.

Proof. By the definition of the free cumulant transform (see (1)) and analytic continuation we have that $\mathcal{C}_{\mu}(z)=z F_{\mu}^{-1}\left(\frac{1}{z}\right)-1$ for all $z$ in $\mathbb{C}^{-}$. Setting $\omega=F_{\mu}^{-1}\left(\frac{1}{z}\right) \in \Omega$, we then get that

$$
\mathcal{C}_{\mu}^{\prime}(z)=F_{\mu}^{-1}\left(\frac{1}{z}\right)-\frac{1}{z}\left(F_{\mu}^{-1}\right)^{\prime}\left(\frac{1}{z}\right)=\omega-\frac{F_{\mu}(\omega)}{F_{\mu}^{\prime}(\omega)} .
$$

Since $F_{\mu}: \Omega \rightarrow \mathbb{C}^{+}$is a bijection, condition (ii) in the lemma is thus equivalent to the condition that $\operatorname{Im}\left(\mathcal{C}_{\mu}^{\prime}(z)\right) \leqslant 0$ for all $z$ in $\mathbb{C}^{-}$. According to Theorem 2.7 the latter condition is, in turn, equivalent to (i) in the lemma.

Theorem 3.3. For any $c$ in $[-1,0]$ the Askey-Wimp-Kerov distribution $\mu_{c}$ is freely selfdecomposable.

Proof. When $c=-1, \mu_{c}$ is a Dirac measure and the theorem is trivial. So let $c$ be a fixed number in $(-1,0]$. According to the proof of Theorem 3.1 in [7] we have that $\mu_{c} \in \mathcal{U I}$, so the reciprocal Cauchy transform $F_{\mu_{c}}$ extends to an analytic bijection $F_{\mu_{c}}: \Omega \rightarrow \mathbb{C}^{+}$defined on some region $\Omega$ containing $\mathbb{C}^{+}$(and depending on $c$ ). According to Lemma 3.2 we then have to establish that

$$
\operatorname{Im}\left(z-\frac{F_{\mu_{c}}(z)}{F_{\mu_{c}}^{\prime}(z)}\right) \leqslant 0
$$

for any $z$ in $\Omega$. We consider first $z$ in $\mathbb{C}^{+}$and observe that

$$
\frac{F_{\mu_{c}}^{\prime}(z)}{F_{\mu_{c}}(z)}=G_{\mu_{c}}(z) \frac{d}{d z}\left(\frac{1}{G_{\mu_{c}}(z)}\right)=-\frac{G_{\mu_{c}}^{\prime}(z)}{G_{\mu_{c}}(z)}=-\frac{d}{d z} \log G_{\mu_{c}}(z),
$$

where log is the standard branch of the logarithm on $\mathbb{C} \backslash\{i y \mid y \geqslant 0\}$. Thus, according to Kerov's Theorem (Theorem 3.1), there exists a probability measure $\tau_{c}$ on $\mathbb{R}$, such that

$$
\frac{F_{\mu_{c}}^{\prime}(z)}{F_{\mu_{c}}(z)}=G_{\tau_{c}}(z), \quad \text { or equivalently } \quad \frac{F_{\mu_{c}}(z)}{F_{\mu_{c}}^{\prime}(z)}=F_{\tau_{c}}(z), \quad\left(z \in \mathbb{C}^{+}\right) .
$$

This implies in particular that

$$
\operatorname{Im}\left(z-\frac{F_{\mu_{c}}(z)}{F_{\mu_{c}}^{\prime}(z)}\right)=\operatorname{Im}\left(z-F_{\tau_{c}}(z)\right)<0, \quad\left(z \in \mathbb{C}^{+}\right),
$$

where the inequality follows from Corollary 5.3 in [9].

Next consider $\omega$ in $\Omega \backslash \mathbb{C}^{+}$. Then according to formula (3.5) in [7] we have that

$$
\frac{F_{\mu_{c}}^{\prime}(\omega)}{F_{\mu_{c}}(\omega)}=\omega-F_{\mu_{c}}(\omega)-\frac{c}{F_{\mu_{c}}(\omega)},
$$

and therefore

$$
\operatorname{Im}\left(\frac{F_{\mu_{c}}^{\prime}(\omega)}{F_{\mu_{c}}(\omega)}\right)=\operatorname{Im}(\omega)-\operatorname{Im}\left(F_{\mu_{c}}(\omega)\right)-c \operatorname{Im}\left(1 / F_{\mu_{c}}(\omega)\right) \leqslant 0,
$$

since $\operatorname{Im}(\omega) \leqslant 0$ and $-c \geqslant 0$, and since $F_{\mu_{c}}(\omega) \in \mathbb{C}^{+}$, so that $1 / F_{\mu_{c}}(\omega) \in \mathbb{C}^{-}$. It follows that

$$
\operatorname{Im}\left(\omega-\frac{F_{\mu_{c}}(\omega)}{F_{\mu_{c}}^{\prime}(\omega)}\right)=\operatorname{Im}(\omega)-\operatorname{Im}\left(\frac{1}{F_{\mu_{c}}^{\prime}(\omega) / F_{\mu_{c}}(\omega)}\right) \leqslant 0,
$$

and this completes the proof. 
Corollary 3.4. For any $\xi$ in $\mathbb{R}$ and $\sigma$ in $(0, \infty)$, the normal distribution $N\left(\xi, \sigma^{2}\right)$ is freely selfdecomposable.

Proof. If $\xi=0$ and $\sigma^{2}=1$, this corresponds to the case $c=0$ in Theorem 3.3. The general case subsequently follows from the fact that $L(\boxplus)$ is closed under scalings and translations.

Remark 3.5. Let $\xi$ and $\sigma$ be a real and a positive number, respectively. For any $t$ in $(0, \infty)$ the probability measure $N\left(\xi, \sigma^{2}\right)^{\boxplus t}$ may be defined as the law at time $t$ of a free Lévy process $\left(X_{t}\right)$ such that $X_{1}$ has law $N\left(\xi, \sigma^{2}\right)$. In particular the free Lévy measure for $N\left(\xi, \sigma^{2}\right)^{\boxplus t}$ is $t \nu$, with $\nu$ the free Lévy measure of $N\left(\xi, \sigma^{2}\right)$, and hence $N\left(\xi, \sigma^{2}\right)^{\boxplus t}$ is FSD as well. In particular this implies that $N\left(\xi, \sigma^{2}\right)^{\boxplus t}$ is unimodal (cf. [17]).

\section{A Proofs of various technical lemmas}

\section{Proof of Lemma 2.1.}

(i) We initially put $\|f\|_{\infty}:=\sup _{x \in[a, b]}|f(x)|<\infty$. For $u$ in $[a, b]$ and $v$ in $(0,1)$ we then have that

$$
\begin{aligned}
\mid \int_{a}^{b} f(x) \log \left((x-u)^{2}+v^{2}\right) d x & -\int_{a}^{b} f(x) \log \left((x-u)^{2}\right) d x \mid \\
& =\left|\int_{a-u}^{0} f(x+u) \log \left(\frac{x^{2}+v^{2}}{x^{2}}\right) d x+\int_{0}^{b-u} f(x+u) \log \left(\frac{x^{2}+v^{2}}{x^{2}}\right) d x\right| \\
& \leqslant \int_{a-b}^{0}\|f\|_{\infty} \log \left(\frac{x^{2}+v^{2}}{x^{2}}\right) d x+\int_{0}^{b-a}\|f\|_{\infty} \log \left(\frac{x^{2}+v^{2}}{x^{2}}\right) d x \\
& =2\|f\|_{\infty} \int_{0}^{b-a} \log \left(\frac{x^{2}+v^{2}}{x^{2}}\right) d x .
\end{aligned}
$$

The resulting expression does not depend on $u$ and tends to 0 as $v \downarrow 0$ by dominated convergence with the dominating function $\log \left(\frac{x^{2}+1}{x^{2}}\right)$ for all $v \in(0,1)$.

(ii) Recall that arg denotes the standard continuous argument function on $\mathbb{C} \backslash\{i y \mid y \leqslant 0\}$, and therefore

$$
\int_{a}^{b} f(x) \arg ((u-x)+i v) d x=\int_{a}^{u} f(x) \arctan \left(\frac{v}{u-x}\right) d x+\int_{u}^{b} f(x)\left(\pi-\arctan \left(\frac{v}{x-u}\right)\right) d x
$$

for any $u$ in $[a, b]$ and $v$ in $[0, \infty)$. Note further that

$$
\left|\int_{a}^{u} f(x) \arctan \left(\frac{v}{u-x}\right) d x\right| \leqslant \int_{0}^{u-a}\|f\|_{\infty}\left|\arctan \left(\frac{v}{y}\right)\right| d y \leqslant\|f\|_{\infty} \int_{0}^{b-a}\left|\arctan \left(\frac{v}{y}\right)\right| d y,
$$

and similarly

$$
\begin{aligned}
\left|\int_{u}^{b} f(x)\left(\pi-\arctan \left(\frac{v}{x-u}\right)\right) d x-\pi(F(b)-F(u))\right| & \leqslant \int_{0}^{b-u}\|f\|_{\infty}\left|\arctan \left(\frac{v}{y}\right)\right| d y \\
& \leqslant\|f\|_{\infty} \int_{0}^{b-a}\left|\arctan \left(\frac{v}{y}\right)\right| d y .
\end{aligned}
$$

Since $\int_{0}^{b-a}\left|\arctan \left(\frac{v}{y}\right)\right| d y$ does not depend on $u$ and converges to 0 , as $v \downarrow 0$, by dominated convergence, the estimates above verify (ii).

(iii) If $0 \notin[a, b]$, then $\sup _{x \in[a, b]}\left|\log \left((u-x)^{2}+v^{2}\right)\right|<\infty$ for $|u+i v|$ small enough, and hence the assertion follows by dominated convergence. We may thus assume in the following that $0 \in[a, b]$, and we establish only that

$$
\int_{0}^{b} f(x) \log \left((u-x)^{2}+v^{2}\right) d x \longrightarrow \int_{0}^{b} f(x) \log \left(x^{2}\right) d x \quad \text { as } u+i v \rightarrow 0 \text { from } \mathbb{C}^{+},
$$


since it follows symmetrically that $\int_{a}^{0} f(x) \log \left((u-x)^{2}+v^{2}\right) d x \rightarrow \int_{a}^{0} f(x) \log \left((u-x)^{2}+v^{2}\right) d x$ as $u+i v \rightarrow 0$ from $\mathbb{C}^{+}$. For $u$ in $(0, \infty)$ we note first that

$$
\begin{aligned}
\mid \int_{0}^{b} f(x) \log \left((x-u)^{2}\right. & \left.+v^{2}\right) d x-\int_{0}^{b} f(x) \log \left(x^{2}\right) d x \mid \\
& =\left|\int_{-u}^{b-u} f(x+u) \log \left(x^{2}+v^{2}\right) d x-\int_{0}^{b} f(x) \log \left(x^{2}\right) d x\right| \\
& \leqslant\left|\int_{-u}^{0} f(x+u) \log \left(x^{2}+v^{2}\right) d x\right|+\left|\int_{0}^{b-u}(f(x+u)-f(x)) \log \left(x^{2}+v^{2}\right) d x\right| \\
& \quad+\left|\int_{0}^{b-u} f(x)\left(\log \left(x^{2}+v^{2}\right)-\log \left(x^{2}\right)\right) d x\right|+\left|\int_{b-u}^{b} f(x) \log \left(x^{2}\right) d x\right| .
\end{aligned}
$$

Assuming henceforth that $u^{2}+v^{2} \leqslant \frac{1}{2} \wedge b$, we have here that

$$
\left|\int_{-u}^{0} f(x+u) \log \left(x^{2}+v^{2}\right) d x\right| \leqslant\|f\|_{\infty} \int_{-u}^{0}-\log \left(x^{2}+v^{2}\right) d x \leqslant-\|f\|_{\infty} \int_{-u}^{0} \log \left(x^{2}\right) d x \longrightarrow 0,
$$

as $u \downarrow 0$, by dominated convergence. Similarly we find that

$$
\left|\int_{b-u}^{b} f(x) \log \left(x^{2}\right) d x\right| \leqslant\|f\|_{\infty} \int_{b-u}^{b}\left|\log \left(x^{2}\right)\right| d x \longrightarrow 0, \quad \text { as } u \downarrow 0 .
$$

We note further that

$$
\begin{aligned}
& \left|\int_{0}^{b-u}(f(x+u)-f(x)) \log \left(x^{2}+v^{2}\right) d x\right| \leqslant\left(\sup _{x \in[0, b-u]}|f(x+u)-f(x)|\right) \int_{0}^{b}\left|\log \left(x^{2}+v^{2}\right)\right| d x \\
& \quad \leqslant\left(\sup _{x \in[0, b-u]}|f(x+u)-f(x)|\right)\left(\int_{0}^{b \wedge \frac{1}{2}}-\log \left(x^{2}\right) d x+\int_{b \wedge \frac{1}{2}}^{b}\left|\log \left(b^{2} \wedge \frac{1}{4}\right)\right| \vee\left|\log \left(b^{2}+\frac{1}{2}\right)\right| d x\right) \\
& \quad \longrightarrow 0, \quad \text { as } u \downarrow 0,
\end{aligned}
$$

since the supremum goes to 0 as $u \downarrow 0$, by uniform continuity of $f$, and since both integrals in the resulting expression are finite. Note finally that

$$
\left|\int_{0}^{b-u} f(x)\left(\log \left(x^{2}+v^{2}\right)-\log \left(x^{2}\right)\right) d x\right| \leqslant\|f\|_{\infty} \int_{0}^{b} \log \left(\frac{x^{2}+v^{2}}{x^{2}}\right) d x \longrightarrow 0, \quad \text { as } v \downarrow 0,
$$

since the integral in the resulting expression goes to 0 as $v \downarrow 0$ as seen in the proof of (i). Combining (22)(26), it follows that $\int_{0}^{b} f(x) \log \left((u-x)^{2}+v^{2}\right) d x \rightarrow \int_{0}^{b} f(x) \log \left(x^{2}\right) d x$ as $u+i v \rightarrow 0$ from $(0, \infty)+i(0, \infty)$. A similar argumentation establishes the same convergence when $u+i v \rightarrow 0$ from $(-\infty, 0)+i(0, \infty)$.

Proof of Lemma 2.3. (i) We must show that $\int_{\mathbb{R}}\left(1 \wedge x^{2}\right) \frac{k(x)}{|x|} d x<\infty$. We note first that

$$
\int_{-1}^{1} x^{2} \frac{k(x)}{|x|} d x=\int_{0}^{1} x k(x) d x+\int_{-1}^{0}|x| k(x) d x
$$

where, by Tonelli's Theorem,

$$
\int_{0}^{1} x k(x) d x=\int_{0}^{1}\left(\int_{x}^{\infty} x \frac{1+y^{2}}{y^{2}} \rho(d y)\right) d x=\int_{0}^{\infty} \frac{1+y^{2}}{y^{2}}\left(\int_{0}^{y \wedge 1} x d x\right) \rho(d y)=\int_{0}^{\infty} \frac{(y \wedge 1)^{2}}{2 y^{2}}\left(1+y^{2}\right) \rho(d y)<\infty,
$$

since $\rho$ is a finite measure, and the function $y \mapsto \frac{(y \wedge 1)^{2}}{2 y^{2}}\left(1+y^{2}\right)$ is bounded on $(0, \infty)$. In the same manner, $\int_{-1}^{0}|x| k(x) d x<\infty$. Note next that

$$
\int_{\mathbb{R} \backslash[-1,1]} \frac{k(x)}{|x|} d x=\int_{1}^{\infty} \frac{k(x)}{x} d x+\int_{-\infty}^{-1} \frac{k(x)}{|x|} d x
$$


where

$\int_{1}^{\infty} \frac{k(x)}{x} d x=\int_{1}^{\infty} \frac{1}{x}\left(\int_{x}^{\infty} \frac{1+y^{2}}{y^{2}} \rho(d y)\right) d x=\int_{1}^{\infty} \frac{1+y^{2}}{y^{2}}\left(\int_{1}^{y} \frac{1}{x} d x\right) \rho(d y)=\int_{1}^{\infty} \log (y) \frac{1+y^{2}}{y^{2}} \rho(d y)<\infty$, since the function $y \mapsto \frac{1+y^{2}}{y^{2}}$ is bounded on $[1, \infty)$, and $\int_{1}^{\infty} \log (y) \rho(d y)<\infty$. In a similar way it follows that $\int_{-\infty}^{-1} \frac{k(x)}{|x|} d x<\infty$, and this completes the proof of (i).

(ii) For any $\varepsilon$ in $(0, \infty)$, there exists $\delta$ in $(0,1)$, such that $\int_{(0, \delta]}\left(1+y^{2}\right) \rho(d y) \leqslant 2 \rho((0, \delta]) \leqslant \varepsilon$. Since $\rho$ is finite we have that $\int_{\delta}^{\infty} y^{-2}\left(1+y^{2}\right) \rho(d y)<\infty$, and we can thus choose $\gamma$ in $(0, \infty)$, such that $\gamma^{2} \int_{\delta}^{\infty} y^{-2}(1+$ $\left.y^{2}\right) \rho(d y) \leqslant \epsilon$. Now for any $x$ in $(0, \delta \wedge \gamma)$ we find that

$$
x^{2} k(x)=x^{2} \int_{x}^{\delta} \frac{1+y^{2}}{y^{2}} \rho(d y)+x^{2} \int_{\delta}^{\infty} \frac{1+y^{2}}{y^{2}} \rho(d y) \leqslant \int_{x}^{\delta} y^{2} \frac{1+y^{2}}{y^{2}} \rho(d y)+\gamma^{2} \int_{\delta}^{\infty} \frac{1+y^{2}}{y^{2}} \rho(d y) \leqslant 2 \epsilon,
$$

and this shows that $x^{2} k(x) \rightarrow 0$ as $x \downarrow 0$. In a similar way, it follows that $x^{2} k(x) \rightarrow 0$ as $x \uparrow 0$, and this completes the proof of (ii).

(iii) For $x$ in $[1, \infty)$ we note first that

$|\log (x) k(x)|=\int_{x}^{\infty} \log (x) \frac{1+y^{2}}{y^{2}} \rho(d y) \leqslant \int_{x}^{\infty} \log (y) \frac{1+y^{2}}{y^{2}} \rho(d y) \leqslant \int_{x}^{\infty} 2 \log (y) \rho(d y) \longrightarrow 0, \quad$ as $x \rightarrow \infty$, since $\int_{1}^{\infty} \log (y) \rho(d y)<\infty$. Similarly it follows that $\log (|x|) k(x) \rightarrow 0$ as $x \rightarrow-\infty$.

(iv) Recall that here log denotes the standard branch of the logarithm on $\mathbb{C} \backslash(-\infty, 0]$, and let arg denote the corresponding argument function. For $z=u+i v$ in $\mathbb{C}^{-}$we then have that

$$
\begin{aligned}
\left|\log (1-z x)+\frac{x z}{1+x^{2}}\right| k(x) & =\left|\frac{1}{2} \log \left((1-u x)^{2}+v^{2} x^{2}\right)+i \arg ((1-u x)+i v x)+\frac{x z}{1+x^{2}}\right| k(x) \\
& \leqslant \frac{1}{2}\left(\log \left(x^{2}\right)+\log \left(\left(x^{-1}-u\right)^{2}+v^{2}\right)\right) k(x)+\left(\pi+\frac{|x z|}{1+x^{2}}\right) k(x),
\end{aligned}
$$

where the resulting expression tends to 0 as $|x| \rightarrow \infty$ by (iii).

By second order Taylor expansion we note next that $\log (1-z x)=-z x-\frac{1}{2} z^{2} x^{2}+o\left(x^{2}\right)$, and therefore

$$
\log (1-z x)+\frac{x z}{1+x^{2}}=-\frac{z x^{3}}{1+x^{2}}-\frac{1}{2} z^{2} x^{2}+o\left(x^{2}\right), \quad \text { as } x \rightarrow 0 .
$$

Consequently,

$$
\left(\log (1-z x)+\frac{x z}{1+x^{2}}\right) k(x)=\left(-\frac{z x}{1+x^{2}}-\frac{1}{2} z^{2}+o(1)\right) x^{2} k(x) \longrightarrow 0, \quad \text { as } x \rightarrow 0,
$$

by (ii). This completes the proof of (iv) and hence the proof of the lemma.

Proof of Lemma 2.4. We consider initially the case $m=1$ and arbitrary $a^{\prime}, b^{\prime}$ such that $a<a^{\prime}<b^{\prime}<b$. It suffices then to show that $G_{f}$ can be extended to a continuous function on $\mathbb{C}^{+} \cup\left(a^{\prime}, b^{\prime}\right)$. For any $z$ in $\mathbb{C}^{+}$ we have that

$$
G_{f}(z)=\int_{a}^{a^{\prime}} \frac{f(x)}{z-x} d x+\int_{a^{\prime}}^{b^{\prime}} \frac{f(x)}{z-x} d x+\int_{b^{\prime}}^{b} \frac{f(x)}{z-x} d x=: G_{1}(z)+G_{2}(z)+G_{3}(z) .
$$

It is clear that $G_{1}$ and $G_{3}$ can be extended to analytic functions on $\mathbb{C}^{+} \cup\left(a^{\prime}, b^{\prime}\right) \cup \mathbb{C}^{-}$, and it remains then to prove that $G_{2}$ can be extended to a continuous function on $\mathbb{C}^{+} \cup\left(a^{\prime}, b^{\prime}\right)$. In the following we denote by $\log$ the standard continuous branch of the logarithm on $\mathbb{C} \backslash\{i y \mid y \leqslant 0\}$. Using integration by parts, we then obtain for $z=u+i v$ in $\mathbb{C}^{+}$that

$$
G_{2}(z)=-f\left(b^{\prime}\right) \log \left(u+i v-b^{\prime}\right)+f\left(a^{\prime}\right) \log \left(u+i v-a^{\prime}\right)+\int_{a^{\prime}}^{b^{\prime}} f^{\prime}(x) \log (u+i v-x) d x
$$


Here the first and second terms $-f\left(b^{\prime}\right) \log \left(u+i v-b^{\prime}\right)+f\left(a^{\prime}\right) \log \left(u+i v-a^{\prime}\right)$ are analytic on $\mathbb{C}^{+} \cup\left(a^{\prime}, b^{\prime}\right)$ with respect to $z=u+i v$. Regarding the integral in (27) an application of Lemma 2.1(i)-(ii) yields that

$$
\begin{aligned}
\int_{a^{\prime}}^{b^{\prime}} f^{\prime}(x) \log (u+i v-x) d x & =\frac{1}{2} \int_{a^{\prime}}^{b^{\prime}} f^{\prime}(x) \log \left((x-u)^{2}+v^{2}\right) d x+i \int_{a^{\prime}}^{b^{\prime}} f^{\prime}(x) \arg (u+i v-x) d x \\
& \longrightarrow \int_{a^{\prime}}^{b^{\prime}} f^{\prime}(x) \log (|x-u|) d x+i \pi\left(f\left(b^{\prime}\right)-f(u)\right)
\end{aligned}
$$

as $v \downarrow 0$, uniformly w.r.t. $u \in\left(a^{\prime}, b^{\prime}\right)$. From this it follows readily that $G_{2}$ can be extended to a continuous function on $\mathbb{C}^{+} \cup\left(a^{\prime}, b^{\prime}\right)$, where

$$
\begin{aligned}
G_{2}(u) & =-f\left(b^{\prime}\right) \log \left(u-b^{\prime}\right)+f\left(a^{\prime}\right) \log \left(u-a^{\prime}\right)+\int_{a^{\prime}}^{b^{\prime}} f^{\prime}(x) \log (|x-u|) d x+i \pi\left(f\left(b^{\prime}\right)-f(u)\right) \\
& =-f\left(b^{\prime}\right) \log \left(b^{\prime}-u\right)+f\left(a^{\prime}\right) \log \left(u-a^{\prime}\right)+\int_{a^{\prime}}^{b^{\prime}} f^{\prime}(x) \log (|x-u|) d x-i \pi f(u)
\end{aligned}
$$

for $u$ in $\left(a^{\prime}, b^{\prime}\right)$.

Suppose next that $m \geqslant 2$, and that $f \in C^{m}((a, b))$. With $a^{\prime}, b^{\prime}$ and $G_{2}$ as above, it suffices to show that the derivatives $G_{2}^{\prime}, G_{2}^{\prime \prime}, \ldots, G_{2}^{(m-1)}$ can be extended to continuous functions on $\mathbb{C}^{+} \cup\left(a^{\prime}, b^{\prime}\right)$. For any $n$ in $\{1, \ldots, m-1\}$ it follows by induction and integration by parts that

$$
G_{2}^{(n)}(z)=\sum_{k=0}^{n-1}(n-1-k) !(-1)^{n-k}\left[\frac{f^{(k)}(x)}{(z-x)^{n-k}}\right]_{x=a^{\prime}}^{x=b^{\prime}}+\int_{a^{\prime}}^{b^{\prime}} \frac{f^{(n)}(x)}{z-x} d x .
$$

From this expression and the preceding part of the proof, it follows readily that $G_{2}^{\prime}, \ldots, G_{2}^{(m-1)}$ can be extended to continuous functions on $\mathbb{C}^{+} \cup\left(a^{\prime}, b^{\prime}\right)$, as desired.

Proof of Lemma 2.6. For each $n$ in $\mathbb{N}$ we introduce first the function $k_{n}^{0}:(0, \infty) \rightarrow[0, \infty)$ defined by

$$
k_{n}^{0}(t)= \begin{cases}k\left(\frac{1}{n}\right), & \text { if } t \in\left(0, \frac{1}{n}\right) \\ k(t), & \text { if } t \in\left[\frac{1}{n}, n\right] \\ 0, & \text { if } t \in(n, \infty),\end{cases}
$$

and we note that $k_{n}^{0} \leqslant k_{n+1}^{0}$ for all $n$. Next we choose a non-negative function $\varphi$ from $C_{c}^{\infty}(\mathbb{R})$, such that $\operatorname{supp}(\varphi) \subseteq[-1,0]$, and $\int_{-1}^{0} \varphi(t) d t=1$. We then define the function $R_{n}:(0, \infty) \rightarrow[0, \infty)$ as the convolution

$$
R_{n}(t)=n \int_{-1 / n}^{0} k_{n}^{0}(t-s) \varphi(n s) d s=\int_{0}^{1} k_{n}^{0}\left(t+\frac{u}{n}\right) \varphi(-u) d u, \quad(t \in(0, \infty)) .
$$

Since $k_{n}^{0}$ is a bounded, decreasing function, it follows immediately from (28) that so is $R_{n}$. Moreover, $\operatorname{supp}\left(R_{n}\right) \subseteq(0, n]$ by the definition of $k_{n}^{0}$. Note also that

$$
R_{n}(t)=n \int_{0}^{n} \varphi(n(t-s)) k_{n}^{0}(s) d s, \quad(t \in(0, \infty)) .
$$

Since $k_{n}^{0}$ as well as the derivatives of $\varphi$ are bounded functions, it follows then by differentiation under the integral sign that $R_{n}$ is a $C^{\infty}$-function on $(0, \infty)$ with bounded derivatives given by

$$
R_{n}^{(p)}(t)=n^{p+1} \int_{0}^{n} \varphi^{(p)}(n(t-s)) k_{n}^{0}(s) d s, \quad(p \in \mathbb{N}, t \in(0, \infty)) .
$$

By dominated convergence it follows further for any $p$ in $\mathbb{N}_{0}$ that

$$
\lim _{t \downarrow 0} R_{n}^{(p)}(t)=n^{p+1} \int_{0}^{n} \varphi^{(p)}(-n s) k_{n}^{0}(s) d s \in \mathbb{R} .
$$


For any $t$ in $(0, \infty)$ and $n$ in $\mathbb{N}$ note next that

$$
R_{n}(t) \leqslant \int_{0}^{1} k_{n+1}^{0}\left(t+\frac{u}{n}\right) \varphi(-u) d u \leqslant \int_{0}^{1} k_{n+1}^{0}\left(t+\frac{u}{n+1}\right) \varphi(-u) d u=R_{n+1}(t) .
$$

Moreover, the monotonicity assumptions imply that $k$ is continuous at almost all $t$ in $(0, \infty)$ (with respect to Lebesgue measure). For such a $t$ we further consider $n$ so large that $t+\frac{u}{n} \in\left[\frac{1}{n}, n\right]$ for all $u$ in $[0,1]$. For such $n$ it follows then that

$$
R_{n}(t)=\int_{0}^{1} k\left(t+\frac{u}{n}\right) \varphi(-u) d u \underset{n \rightarrow \infty}{\longrightarrow} \int_{0}^{1} k(t) \varphi(-u) d u=k(t)
$$

by monotone convergence. We conclude that $R_{n}(t) \uparrow k(t)$ as $n \rightarrow \infty$ for almost all $t$ in $(0, \infty)$.

Applying the considerations above to the function $\kappa:(0, \infty) \rightarrow[0, \infty)$ given by $\kappa(t)=k(-t)$, it follows that we can construct a sequence $\left(L_{n}\right)_{n \in \mathbb{N}}$ of non-negative functions defined on $(-\infty, 0)$ and with the following properties:

- For all $n$ in $\mathbb{N}$ the function $L_{n}$ has bounded support.

- For all $n$ in $\mathbb{N}$ we have that $L_{n} \in C^{\infty}((-\infty, 0))$, and $L_{n}^{(p)}$ is bounded for all $p$ in $\mathbb{N}_{0}$.

- For all $n$ in $\mathbb{N}$ the function $L_{n}$ is increasing on $(-\infty, 0)$.

- $L_{n}(t) \uparrow k(t)$ as $n \rightarrow \infty$ for almost all $t$ in $(-\infty, 0)$ (with respect to Lebesgue measure).

We are now ready to define $k_{n}: \mathbb{R} \backslash\{0\} \rightarrow[0, \infty)$ by

$$
k_{n}(t)= \begin{cases}R_{n}(t), & \text { if } t>0, \\ L_{n}(t), & \text { if } t<0 .\end{cases}
$$

It is then apparent from the argumentation above that $k_{n}$ satisfies the conditions (a)-(c) in the lemma, and it remains to show that $\frac{|t| k_{n}(t)}{1+t^{2}} d t \rightarrow \frac{|t| k(t)}{1+t^{2}} d t$ weakly as $n \rightarrow \infty$. But for any bounded continuous function $g: \mathbb{R} \rightarrow \mathbb{R}$ we find that

$$
\begin{aligned}
\int_{\mathbb{R}} g(t) \frac{|t| k_{n}(t)}{1+t^{2}} d t & =\int_{-\infty}^{0} g(t) \frac{|t| L_{n}(t)}{1+t^{2}} d t+\int_{0}^{\infty} g(t) \frac{t R_{n}(t)}{1+t^{2}} d t=\int_{-\infty}^{0} g(t) \frac{|t| L_{n}(t)}{1+t^{2}} d t+\int_{0}^{\infty} g(t) \frac{t R_{n}(t)}{1+t^{2}} d t \\
& \underset{n \rightarrow \infty}{\longrightarrow} \int_{-\infty}^{0} g(t) \frac{|t| k(t)}{1+t^{2}} d t+\int_{0}^{\infty} g(t) \frac{t k(t)}{1+t^{2}} d t=\int_{\mathbb{R}} g(t) \frac{|t| k(t)}{1+t^{2}} d t
\end{aligned}
$$

where, when letting $n \rightarrow \infty$, we used dominated convergence on each of the integrals; note in particular that $\frac{|t| L_{n}(t)}{1+t^{2}}$ and $\frac{t R_{n}(t)}{1+t^{2}}$ are dominated almost everywhere by $\frac{|t| k(t)}{1+t^{2}}$ on the relevant intervals, and here $\int_{\mathbb{R}} \frac{|t| k(t)}{1+t^{2}} d t<\infty$, since $\frac{k(t)}{|t|} d t$ is a Lévy measure. This completes the proof.

\section{Acknowledgments}

T. Hasebe was supported by JSPS Grant-in-Aid for Young Scientists (B) 15K17549. N. Sakuma was supported by JSPS Grant-in-Aid for Scientific Research (C) 15K04923. Part of this work was done while the second author was visiting The University of Aarhus. He sincerely appreciates the hospitality of The University of Aarhus. The authors are grateful to Octavio Arizmendi for a useful suggestion. 


\section{References}

[1] Akhiezer, Naum I. The Classical Moment Problem. Oliver \& Boyd Ltd (1965).

[2] Anshelevich, Michael. Free martingale polynomials. J. Funct. Anal. 201 (2003), no. 1, 228-261.

[3] Askey, Richard and Wimp, Jet. Associated Laguerre and Hermite polynomials. Proc. Royal Soc. Edinburgh 96A (1984), 15-37.

[4] Arizmendi, Octavio and Hasebe, Takahiro. On a class of explicit Cauchy-Stieltjes transforms related to monotone stable and free Poisson laws. Bernoulli 19 (2013), no. 5B, 2750-2767.

[5] Barndorff-Nielsen, Ole E. and Thorbjørnsen, Steen. Self-decomposability and Lévy processes in free probability. Bernoulli 8 (2002), no. 3, 323-366.

[6] Barndorff-Nielsen, Ole E. and Thorbjørnsen, Steen. A connection between free and classical infinite divisibility. Infin. Dimens. Anal. Quantum Probab. Relat. Top. 7 (2004), no. 4, 573-590.

[7] Belinschi, Serban T., Bożejko, Marek, Lehner, Franz and Speicher, Roland. The normal distribution is $\boxplus$-infinitely divisible. Adv. Math. 226 (2011), no. 4, 3677-3698.

[8] Benaych-Georges, Florent. Taylor expansions of R-transforms: application to supports and moments. Indiana Univ. Math. J. 55 (2006), no. 2, 465-481.

[9] Bercovici, Hari and Voiculescu, Dan. Free convolution of measures with unbounded support. Indiana Univ. Math. J. 42 (1993), no. 3, 733-773.

[10] Bercovici, Hari and Pata, Vittorino. Stable laws and domains of attraction in free probability theory. With an appendix by Philippe Biane. Ann. of Math. (2) 149 (1999), no. 3, 1023-1060.

[11] Bożejko, Marek and Bryc, Włodzimierz. On a class of free Lévy laws related to a regression problem. J. Funct. Anal. 236 (2006), no. 1, 59-77.

[12] Chistyakov, Gennadii P. and Goetze, Friedrich. Limit theorems in free probability theory. I. Ann. Probab. 36, no. 1 (2008), 54-90.

[13] Feller, William. An introduction to probabililty theory and its applications II, second edition. Wiley Series in Probability and Mathematical Statistics, John Wiley \& Sons, New York (1971).

[14] Gnedenko, Boris Vladimirovich and Kolmogorov, Andrei Nikolaevich. Limit Distributions for Sums of Independent Random Variables. Addison-Wesley Publishing Company, Inc., Reading, Mass.-LondonDon Mills., Ont. (1968).

[15] Haagerup, Uffe and Thorbjørnsen, Steen. On the free gamma distributions. Indiana Univ. Math. J. 63 (2014), no. 4, 1159-1194.

[16] Hasebe, Takahiro and Sakuma, Noriyoshi. Unimodality for free Lévy processes. Ann. Inst. Henri Poincaré Probab. Stat. 53 (2017), no. 2, 916-936. 
[17] Hasebe, Takahiro and Thorbjørnsen, Steen. Unimodality of the freely selfdecomposable probability laws. J. Theoret. Probab. 29 (2016), no. 3, 922-940.

[18] Kerov, Sergei. Interlacing measures. In: Kirillovs Seminar on Representation Theory, Amer. Math. Soc. Transl. vol. 181, Amer. Math. Soc. (1998), pp. 35-83.

[19] Nica, Alexandru and Speicher, Roland. Lectures on the combinatorics of free probability. London Mathematical Society Lecture Note Series, 335. Cambridge University Press, Cambridge, 2006. $\mathrm{xvi}+417$

[20] Pérez-Abreu, Víctor and Sakuma, Noriyoshi. Free generalized gamma convolutions. Electron. Commun. Probab. 13 (2008), 526-539.

[21] Saitoh, Naoko and Yoshida, Hiroaki. The infinite divisibility and orthogonal polynomials with a constant recursion formula in free probability theory. Probab. Math. Statist. 21 (2001), no. 1, Acta Univ. Wratislav. No. 2298, 159-170.

[22] Sato, Ken-iti. Lévy processes and infinitely divisible distributions. Translated from the 1990 Japanese original. Revised by the author. Cambridge Studies in Advanced Mathematics, 68. Cambridge University Press, Cambridge, 1999. xii+486 pp.

[23] Voiculescu, Dan, Dykema, Ken and Nica, Alexandru. Free random variables. A noncommutative probability approach to free products with applications to random matrices, operator algebras and harmonic analysis on free groups. CRM Monograph Series, 1. American Mathematical Society, Providence, RI, 1992. vi+70 pp. 